\title{
Superconductivity and Dirac Fermions in 112-phase Pnictides
}

\author{
S. J. Ray* \\ Department of Physics, Indian Institute of Technology Patna, Bihta 801103, India and \\ Institute of Materials Science, Technische Universität Darmstadt, \\ Alarich-Weiss-Straße 2, Darmstadt 64287, Germany \\ L. Alff \\ Institute of Materials Science, Technische Universität Darmstadt, \\ Alarich-Weiss-Straße 2, Darmstadt 64287, Germany
}

\begin{abstract}
This article reviews the status of current research on the 112-phase of pnictides. The 112-phase has gained augmented attention due to the recent discovery of high-temperature superconductivity in $\mathrm{Ca}_{1-\mathrm{x}} \mathrm{La}_{\mathrm{x}} \mathrm{FeAs}_{2}$ with a maximum critical temperature $T_{\mathrm{c}} \sim 47 \mathrm{~K}$ upon $\mathrm{Sb}$ substitution. The structural, magnetic, and electronic properties of $\mathrm{Ca}_{1-\mathrm{x}} \mathrm{La}_{\mathrm{x}} \mathrm{FeAs}_{2}$ bear some similarities with other superconducting pnictide phases, however, the different valence states of the pnictogen and the presence of a metallic spacer layer are unique features of the 112-system. Low-temperature superconductivity which coexists with antiferromagnetic order was observed in transition metal $(\mathrm{Ni}$, $\mathrm{Pd}$ ) deficient 112-compounds like $\mathrm{CeNi}_{\mathrm{x}} \mathrm{Bi}_{2}, \mathrm{LaPd}_{\mathrm{x}} \mathrm{Bi}_{2}, \mathrm{LaPd}_{\mathrm{x}} \mathrm{Sb}_{2}, \mathrm{LaNi}_{\mathrm{x}} \mathrm{Sb}_{2}$. Besides superconductivity, the presence of naturally occurring anisotropic Dirac Fermionic states were observed in the layered 112-compounds $\mathrm{SrMnBi}_{2}, \mathrm{CaMnBi}_{2}, \mathrm{LaAgBi}_{2}$ which are of significant interest for future nanoelectronics as an alternative to graphene. In these compounds, the linear energy dispersion resulted in a high magnetoresistance that stayed unsaturated even at the highest applied magnetic fields. Here, we describe various 112-type materials systems combining experimental results and theoretical predictions to stimulate further research on this less well-known member of the pnictide family.
\end{abstract}

\section{Contents}

\section{Introduction}

II. Synthesis Techniques

A. Bulk Synthesis

III. Crystal Structure

IV. Electronic Structure

A. Theoretical Investigations

B. Experimental Results

V. Magnetic Structure

VI. Superconducting Properties

A. Low Critical Temperature Materials

B. High Critical Temperature Materials

1. Rare Earth Substitution

2. Transition Metal Substitution

3. Critical Current and Critical Field

4. Effect of Pressure

VII. Dirac Fermions

VIII. Thin Films

A. Growth by Molecular Beam Epitaxy Technique

B. Thin Film Structure

C. Superconducting and Magnetic Properties of Thin Films

IX. Conclusion and outlook

References

*Corresponding author : ray@iitp.ac.in, ray.sjr@gmail.com

\section{Introduction}

1

2

2

3

5

5

6

6

7

7

7

7

9

10

10

11

12

13

13

13

14

15
The discovery of superconductivity in the pnictide family of superconductors $\mathrm{LaOFeP}[1]$ has fuelled research on the Fe-based superconducting compounds after the observation of a critical temperature of $T_{\mathrm{c}} \sim 26 \mathrm{~K}$ in the isostructural compound $\mathrm{La}\left(\mathrm{O}_{1-x} \mathrm{~F}_{x}\right) \mathrm{FeAs}$ [2], which was soon increased up to $43 \mathrm{~K}$ [3] under the application of high pressure [4]. Very soon, an even higher $T_{\mathrm{c}}$ of $54 \mathrm{~K}$ was observed in $\mathrm{SmFeAsO}_{1-x} \mathrm{~F}_{x}[5,6]$. With different levels of doping and elemental substitutions $T_{\mathrm{c}}$ values of $55-58 \mathrm{~K}$ [7-10] were observed in several other compounds in the 1111-family of pnictides. In the next few years, superconductivity was found in various other Fe-based pnictide systems like (Ba,K) $\mathrm{Fe}_{2} \mathrm{As}_{2}$ (122-type) [11], FeSe (11-type) [12], LiFeAs (111-type) [13-15] and, most recently, $\mathrm{Ca}_{1-\mathrm{x}} \mathrm{La}_{\mathrm{x}} \mathrm{FeAs}_{2}$ (112-type) with a $T_{\mathrm{c}}$ of $38 \mathrm{~K}$ [1618]. The fundamental interest in these materials lies in understanding the mechanism behind the coexistence of superconductivity and magnetism. The high critical fields and isotropic critical currents [19-21] could be of interest for electrical power and magnetic applications. Apart from the presence of Fe in these compounds, which is believed to be harmful for conventional superconductivity, the uniqueness lies in the origin of superconductivity due to the presence of the Fe-3d electrons[4].

Although there are several structurally different phases of pnictides, they all contain a common Fe-pnictogen $(P n)$ layer in a tetrahedral arrangement that is separated by blocking layers. The composition of the blocking layer is believed to affect the superconducting properties [22]. The Fe- $P n$ (or Fe- $C h$ ) layers are tetrahedrally coordi- 
nated by the $P n$ or $C h$ (chalcogenide) atoms, and highest critical temperatures were observed for an ideal tetrahedral arrangement [23] with the Fe-As-Fe bond angle closest to $109.47^{\circ}$. Similar to the high- $T_{c}$ Cuprates, the pnictides are also quasi-two-dimensional with reduced electronic coupling along the $c$-axis, and the appearance of superconductivity is observed upon the suppression of the antiferromagnetic order [24]. In comparison to Cuprates, however, the range of superconducting materials is much larger in case of the pnictides offering a huge range of chemical substitution possibilities [25]. Due to the limited availability of high-quality and larger sized single crystals [22], research was focussed on the 1111- and 122-type compounds. Recently, significant interest has shifted to 11-type FeSe thin films[26], where evidence of strain induced interfacial superconductivity was observed up to a maximum $T_{\mathrm{c}} \sim 109 \mathrm{~K}[27]$.

A prediction of the existence of a 112-type pnictide phase was made by Shim et al. [28] in the hypothetical compounds $\mathrm{BaFeAs}_{2}$ and $\mathrm{BaFeSb}_{2}$ with metallic blocking layers unlike other pnictide systems where these layers are insulating. While these compounds remain elusive, the related compound $\mathrm{SrMnBi}_{2}$ was synthesized [29-31] as single crystals with physical and structural similarities to $\mathrm{BaFeAs}_{2}$. Despite having a large Néel temperature $\left(T_{\mathrm{N}} \sim 290 \mathrm{~K}\right)$, no evidence of superconductivity was observed in $\mathrm{SrMnBi}_{2}$. Band structure calculations suggested the presence of anisotropic Dirac fermions in the Bi square net layer which was experimentally confirmed later through the observation of quantum oscillations and angle-resolved photoelectron spectroscopy (ARPES) measurements [31].

Superconductivity in the 112-type pnictide was first reported in the CeTMPn $n_{2}(T M=$ transition metal, $P n=$ pnictogen) family of intermetallic compounds with $T_{\mathrm{N}} \sim$ $5 \mathrm{~K}$ although several heavy-fermion superconductors with layered tetragonal structures were known earlier [32]. Superconductivity in $\mathrm{CeNi}_{\mathrm{x}} \mathrm{Bi}_{2}$ with $T_{\mathrm{c}} \sim 4.2 \mathrm{~K}[33,34]$ was claimed to originate from $\mathrm{Ni}$ deficiency, as no evidence of bulk superconductivity was observed in the parent compound $\mathrm{CeNiBi}_{2}$ [35-37]. However, it has been suggested from the coexistence of light and heavy carriers that the superconducting charge carriers are hosted by the pnictogen square net layer [33]. The presence of superconductivity with low superconducting volume fraction (SVF) were also observed in $\mathrm{LaNi}_{x} \mathrm{Bi}_{2}(\mathrm{SVF}=1-3 \%)[38,39]$, $\mathrm{NdNi}_{x} \mathrm{Bi}_{2}(\mathrm{SVF}=14 \%)$ [33], and $\mathrm{YNi}_{x} \mathrm{Bi}_{2}$ [33] $(\mathrm{SVF}=$ $17 \%$ ) [33].

Interest in the 112-system has grown considerably after the discovery of a high $T_{\mathrm{c}}$ of $34 \mathrm{~K}$ in $\mathrm{Ca}_{1-\mathrm{x}} \mathrm{La}_{\mathrm{x}} \mathrm{FeAs}_{2}$ [16] and in $\mathrm{Ca}_{1-\mathrm{x}} \mathrm{Pr}_{\mathrm{x}} \mathrm{FeAs}_{2}$ with $T_{\mathrm{c}} \sim 20 \mathrm{~K}$ [40]. Subsequently, it was reported that adding a small amount of $\mathrm{P}(0.5 \%)$ and $\mathrm{Sb}(1 \%)$ substituting As in the parent compound leads to a drastic enhancement of $T_{\mathrm{c}}$ in $\mathrm{Ca}_{1-\mathrm{x}} \mathrm{La}_{\mathrm{x}} \mathrm{FeAs}_{2}$ to $41 \mathrm{~K}$ and $43 \mathrm{~K}$, respectively [17]. Later, it was revealed that a larger level of $\mathrm{Sb}$ doping can further enhance $T_{c}$ to $47 \mathrm{~K}$ in $\mathrm{Ca}_{1-x} \mathrm{La}_{x} \mathrm{Fe}\left(\mathrm{As}_{1-y} \mathrm{Sb}_{y}\right)$ [18] which is higher than the maximum $T_{\mathrm{c}}$ observed in bulk 122, 11, 111-type pnictides so far. One interesting fact about the superconductivity in the 112-phase is the existence of mixed-valance states of the pnictogen [33, 38] which has different valencies in the $P n$ square net layer and the metal-pnictogen layers.

In the past two years, research interest again increased for $\mathrm{Ca}_{1-\mathrm{x}} \mathrm{La}_{\mathrm{x}} \mathrm{FeAs}_{2}$ [41-54] due to the availability of single crystals, high- $T_{\mathrm{c}}$ in the bulk phase, and the possibility of a range of substitutions. Significant amount of research work was done earlier in other 112-type materials like $\mathrm{LaPd}_{x} \mathrm{Sb}_{2}$ [55], $\mathrm{LaPd}_{x} \mathrm{Bi}_{2}[55,56], \mathrm{CeNi}_{x} \mathrm{Sb}_{2}$ $[33,34,36], \mathrm{LaNi}_{x} \mathrm{Sb}_{2}[57,58]$ etc. with low $T_{\mathrm{c}} \sim<$ $5 \mathrm{~K}$. Although primary research work in the 112 -system started to look for possible high- $T_{\mathrm{c}}$ materials, recently a large amount of work has also been performed with respect to anisotropic Dirac fermionic states[29-31, 59$64]$ in $(\mathrm{Ca} / \mathrm{Sr}) \mathrm{MnSb}_{2}[61,63,65,66], \mathrm{LaAgBi}_{2}[60,67-$ 69] and understanding their role in the observed large magnetoresistance $[30,65,66,68,70,71]$ and magnetothermopower generation $[60,71]$. The purpose of this review article is to summarise the work done in the 112systems so far, and to stimulate further research.

\section{Synthesis Techniques}

Similar to the other pnictide systems, primarily three techniques are used for bulk sample preparation of 112pnictides[25]: (a) solid state reaction, (b) high-pressure synthesis, and (c) self-flux method. The first two methods are mostly used for polycrystalline and powder samples, while the last one is convenient for single crystal growth. Growth of single crystalline thin films by molecular beam epitxy (MBE) is discussed in detail in Sec. VIII A.

\section{A. Bulk Synthesis}

The parent compound $\mathrm{CaFeAs}_{2}$ has not yet been synthesised, but incorporation of a small amount of La in place of $\mathrm{Ca}[16,17]$ was found to be essential for stabilising the 112-phase and inducing superconductivity. Single crystals of $\mathrm{Ca}_{1-\mathrm{x}} \mathrm{La}_{\mathrm{x}} \mathrm{FeAs}_{2}$ were grown using FeAs-selfflux [16-18, 43, 49, 51] where all the constituents Ca, La, As, FeAs were mixed in appropriate stoichiometric ratio inside an aluminium crucible and sealed inside an evacuated quartz tube. In order to avoid contamination from the atmosphere, the whole process was carried out inside a glove box filled with argon gas. The sealed ampules were heated at appropriate temperatures typically at a maximum of $1100^{\circ} \mathrm{C}$ and kept there for several hours. Finally the furnace was cooled slowly $\left(\sim 1.25^{\circ} \mathrm{C} /\right.$ hour $)$ to room temperature before taking the single crystals of maximum size of $2 \mathrm{~mm}$ [45] out of the furnace. Zhou et al.[45, 47] suggested that larger amounts of starting materials are necessary for the growth of large sized crystals and a small amount of $\mathrm{CaO}$ is helpful for crystallisation.

Polycrystalline samples were synthesised using solid state reaction inside a high-pressure cell [40, 48]. For 
a

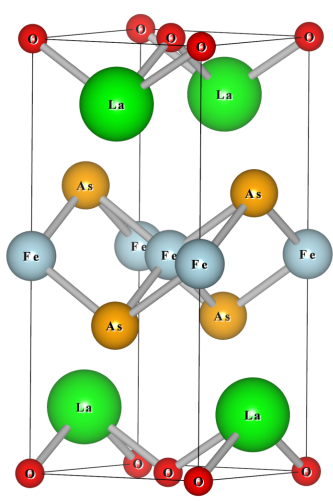

LaFeAsO b

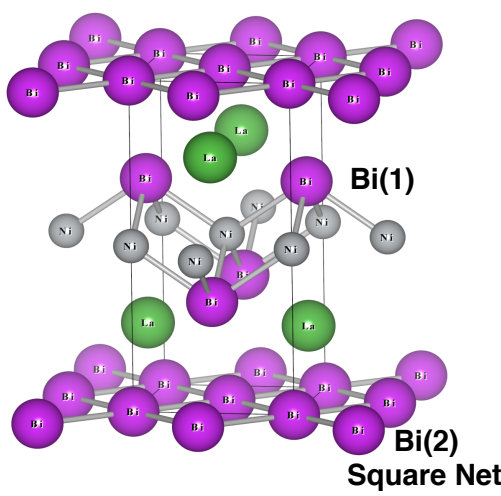

$\mathrm{LaNi}_{\mathbf{x}} \mathrm{Bi}_{2}$ c

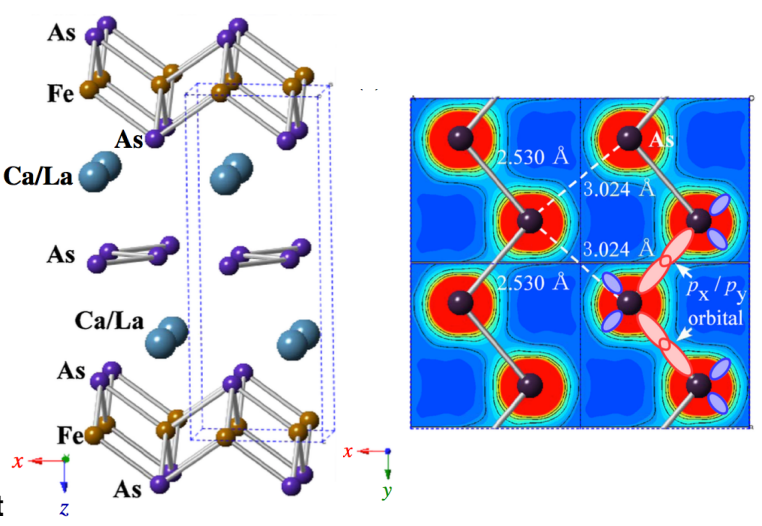

$\mathrm{Ca}_{1-\mathrm{x}} \mathrm{La}_{\mathrm{x}} \mathrm{FeAs} \mathrm{s}_{2}$

FIG. 1: Schematic crystal structures of (a) 1111-type LaFeAsO, (b) 112-type $\mathrm{LaNi}_{\mathrm{x}} \mathrm{Bi}_{2}$ and (c) 112-type Ca $\mathrm{Ca}_{1-\mathrm{x}} \mathrm{La}_{\mathrm{x}} \mathrm{FeAs} 2$ [16]. (d) Top view of the As-zigzag chains in $\mathrm{Ca}_{1-\mathrm{x}} \mathrm{La}_{\mathrm{x}} \mathrm{FeAs}_{2}$. The colour map represents the contour of the charge distribution around As-atoms. Charge accumulation between neighbouring As-atoms are suggestive of the formation of the covalent bonds[4, 16]. [Fig. 1(c-d) : Reprinted with permission from Katayama et al.[16]. Copyright 2013 by the Physics Society of Japan.]

$\mathrm{Ca}_{1-x} R E_{x} \mathrm{FeAs}_{2}(R E=$ rare earths from La $\rightarrow \mathrm{Gd})$ synthesis [48], a mixture of FeAs, REAs, Ca and As powders were mixed and pelletised which was later allowed to react inside a boron nitride crucible between $1000-1200^{\circ} \mathrm{C}$ for $1 \mathrm{~h}$ under $2 \mathrm{GPa}$ pressure [42, 48, 72]. Sala et al. [48] pointed out that high pressure for synthesis is essential to incorporate smaller $R E$-ions and at much higher pressure $(>2 \mathrm{GPa})$ doping of $\mathrm{Tb}, \mathrm{Dy}, \mathrm{Ho}$ and $\mathrm{Y}$ into the 112-phase could be possible.

Single crystal synthesis of $\mathrm{CeNi}_{\mathrm{X}} \mathrm{Bi}_{2}[37]$, $\mathrm{CePd}_{1-x} \mathrm{Bi}_{2}[73], \quad \mathrm{LaPd}_{1-x} \mathrm{Bi}_{2}$ [56], and $\mathrm{SrMnBi}_{2}$ [29] needed excess $\mathrm{Bi}$ flux and different temperature treatments while polycrystalline samples [33, 36, 38] were prepared inside an evacuated silica tube through standard solid state reaction at elevated temperatures for $R E \mathrm{Ni}_{x} \mathrm{Bi}_{2}(R E=\mathrm{La}, \mathrm{Ce}, \mathrm{Nd}, \mathrm{Y})$. It was observed that the $\mathrm{Bi}$ and $\mathrm{Sb}$-based 112-systems decompose gradually when exposed to ambient atmosphere. Hence, storage of these materials in evacuated atmosphere is essential for achieving longer lifetime[33, 34, 36, 55, 57].

\section{Crystal Structure}

Initial reports on 112-type pnictide systems were made on $R E \mathrm{Ni}_{x} \mathrm{Bi}_{2}[33]$ where $R E=\mathrm{La}, \mathrm{Ce}, \mathrm{Nd}, \mathrm{Gd}$ and other rare earth elements. These 112-compounds crystallise in the $\mathrm{HfCuSi}_{2}$-type structure, which can be related to 1111-type compounds with the $\mathrm{ZrCuSiAs-type}$ structure. Structural similarity between the two phases can be found from Fig 1(a-b). The 1111-compound $\mathrm{LaFeAsO}[2]$ contains two different anions (O/As) while the 112 -compound $R E \mathrm{Ni}_{x} \mathrm{Bi}_{2}$ has only $\mathrm{Bi}$ as anion, but in two different valance states, namely $\mathrm{Bi}(1)$ and $\mathrm{Bi}(2)$.
In the stoichiometrically deficient $\mathrm{Ni}_{x} \mathrm{Bi}$ layer (analogous to the FeAs layer in LaFeAsO), Bi(1) forms a distorted tetrahedron in a trivalent charge state due to Coulomb attraction. This forces the other $\mathrm{Bi}(2)$ ion in a charge state of -1 to occupy a narrower space with a shorter BiBi bond-length to form a square net layer as illustrated in Fig. 1(b). The presence of the two-dimensional square net layer is the most unique feature of the 112-phase which stabilises due to the Coulomb attraction driven relaxation between the $R E$-ions. The square net layer can be considered as the blocking layer of the 112-system, though it is metallic unlike the insulating blocking layers present in other pnictide phases. The presence of the two different oxidation states of the pnictide was observed by XPS in $\mathrm{LaPd}_{\mathrm{x}} \mathrm{Sb}_{2}$ [55] where two $3 d$ photoelectron lines of $\mathrm{Sb}$ are separated by $\Delta E=9.4 \mathrm{eV}$ that correspond to -1 and -3 oxidation states of the Sb-atoms. A similar metallic square net layer was also observed in $\mathrm{SrMnBi}_{2}$ (crystallizes in the $\mathrm{SrZnBi}_{2}$-type structure with SG $I 4 / \mathrm{mmm}$ (no. 139) confirmed via neutron scattering measurements[63] as opposed to $\mathrm{LaPd}_{x} \mathrm{Pn}_{2}$ with $\mathrm{P} 4 / \mathrm{nmm}$ (no. 129) symmetry) which is metallic with a large $T_{\mathrm{N}} \sim 290 \mathrm{~K}[29]$. Multiple Dirac cone like dispersions were observed close to the Fermi level. In the single crystalline $R \mathrm{Ni}_{x} \mathrm{Bi}_{2 \pm y}$ (for $R=\mathrm{La}, \mathrm{Ce}-\mathrm{Nd}$, Sm, Gd-Dy), a monotonic decrease in the lattice parameters $a$ $(2.1 \%)$ and $c(5.3 \%)$ were observed due to the lanthanide contraction[39].

Initial reports suggests that the 112-type $\mathrm{Ca}_{1-\mathrm{x}} \mathrm{La}_{\mathrm{x}} \mathrm{FeAs}_{2}$ crystallises in monoclinic structure with space group $\mathrm{P} 22_{1}$ (no. 4)[16] or $\mathrm{P} 2_{1} / m$ (no. 11)[40, 48] which is different from the other pnictide systems having 
tetragonal or orthorhombic space groups. However, recently Harter et al.[74] observed second harmonic generation (SHG) in $\mathrm{Ca}_{1-\mathrm{x}} \mathrm{La}_{\mathrm{x}} \mathrm{FeAs}_{2}$ that is a signature of noncentrosymmetric crystal structure, which suggets that the space group of $\mathrm{Ca}_{1-\mathrm{x}} \mathrm{La}_{\mathrm{x}} \mathrm{FeAs}_{2}$ should not be centrosymmetric $\mathrm{P} 2{ }_{1} / m$, but noncentrosymmetric $\mathrm{P} 2{ }_{1}$. The monoclinic structure stays stable up to $450 \mathrm{~K}[16]$. Alternatively stacked FeAs layers are present in $\mathrm{Ca}_{1-\mathrm{x}} \mathrm{La}_{\mathrm{x}} \mathrm{FeAs}_{2}$ (Fig. 1(c)) separated by zig-zag As bond layers with $\mathrm{Ca} / \mathrm{La}$ placed in between them [4]. The distance between neighbouring FeAs layers is slightly larger than in the 1111-phase materials. The most interesting feature of this material is the presence of $2 \mathrm{D}$ As layers with two different bond lengths that were measured using synchrotron X-ray diffraction [16] as shown in Fig. $1(\mathrm{~d})$. The shorter one $(\sim 2.53 \AA)$ is identical to the As-As single bond length with $\mathrm{As}^{-}$( $4 p^{4}$ configuration) forming a one-dimensional zig-zag chain along the $b$-axis, while the larger one $(\sim 3.02 \AA)$ corresponds to the interchain distance between the zig-zag chains. In the FeAs layer, $\mathrm{As}$ is in a $\mathrm{As}^{3-}$ valance state ( $4 p^{6}$ configuration). The chemical formula of $\mathrm{Ca}_{1-\mathrm{x}} \mathrm{La}_{\mathrm{x}} \mathrm{FeAs}_{2}$ can be written as $\left(\mathrm{Ca}_{1-x}^{2+} R E_{x}^{3+}\right)\left(\mathrm{Fe}^{2+} \mathrm{As}^{3-}\right) \mathrm{As}^{-} \cdot x e^{-}$with excess charge injected inside the FeAs layer [4]. This can be compared to the 1111-type CaFeAsF whose structure can be written similarly as $\left(\mathrm{Ca}_{1-x}^{2+} \mathrm{RE}_{x}^{3+}\right)\left(\mathrm{Fe}^{2+} \mathrm{As}^{3-}\right) \mathrm{F}^{-} \cdot x e^{-}$ where $\mathrm{F}^{-}$forms a square network. In this way, the $112-\mathrm{Ca}_{1-\mathrm{x}} \mathrm{La}_{\mathrm{x}} \mathrm{FeAs} \mathrm{s}_{2}$ structure can be related to 1111$\mathrm{CaFeAsF}$, although the chemical bondings in the spacer layers are not the same in both cases. For CaFeAsF, the $\mathrm{CaF}$ layer is made of strong ionic bonds while CaAs layers in $\mathrm{Ca}_{1-\mathrm{x}} \mathrm{La}_{\mathrm{x}} \mathrm{FeAs}_{2}$ consists of zig-zag As chains of covalent bonds weakly coupled to the adjacent Ca layers. For this reason, the interlayer distance between FeAs planes in $112-\mathrm{Ca}_{1-\mathrm{x}} \mathrm{La}_{\mathrm{x}} \mathrm{FeAs}_{2}(\sim 10.35 \AA)$ [18] is higher than in CaFeAsF $(\sim 8.6 \AA)$ [75]. This layered structure was confirmed by high-angle annular dark field-scanning transmission electron microscopy (HAADF-STEM) measurements of ( $\mathrm{Ca}, \mathrm{Pr}) \mathrm{FeAs}_{2}$ [40] with an interlayer distance of $10.4 \AA$. X-ray scattering data[76] suggested that microscopic manipulation of the electronically active FeAs layer is more effective compared to the larger structural tuning for controlling the superconducting properties of $\mathrm{Ca}_{1-\mathrm{x}} \mathrm{La}_{\mathrm{x}} \mathrm{FeAs}_{2}$.

Recently Joseph et al. [44] reported the structural evolution of $\mathrm{Ca}_{1-\mathrm{x}} \mathrm{La}_{\mathrm{x}} \mathrm{FeAs}_{2}(x=0.18)$ with temperature $(110 \mathrm{~K}-300 \mathrm{~K})$ from powdered X-ray diffraction measurements. The expansion of the $c$-axis lattice constant goes through a distinct change around $150 \mathrm{~K}$ which can be correlated with a change in the resistivity slope. In this temperature range, the in-plane lattice constants go through a constant thermal expansion which is an order of magnitude smaller $\left(\sim 0.3 \times 10^{-4} \AA / \mathrm{K}\right)$ than for the $c$-lattice constant. The anisotropic thermal expansion suggests a change in the inter-layer interaction with temperature, although its relation to the resistivity change with temperature is not clear yet.

Alternative claims have been made that instead of

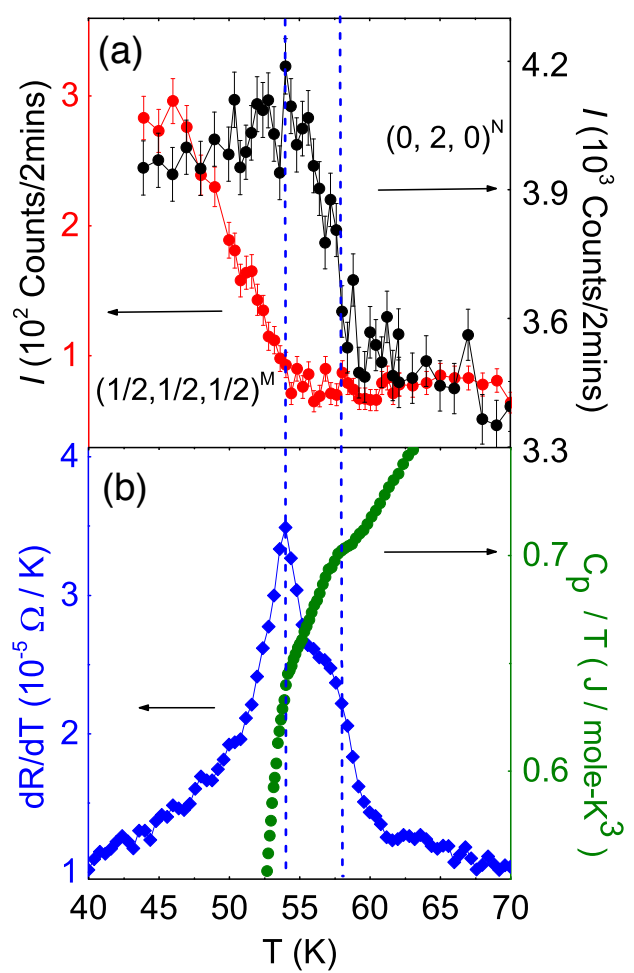

FIG. 2: Temperature dependence of the (a) neutron intensity of nuclear $\left(\begin{array}{lll}0 & 2 & 0\end{array}\right)^{N}$ and magnetic $\left(\begin{array}{llll}1 / 2 & 1 / 2 & 1 / 2\end{array}\right)^{M}$ peaks, (b) Specific heat $\left(C_{p}\right)$ and derivative of the in-plane resistivity $(\rho \| a b)$ in $\mathrm{Ca}_{1-\mathrm{x}} \mathrm{La}_{\mathrm{x}} \mathrm{FeAs}_{2}$. The dotted lines are the temperatures at which the structural resp. magnetic phase transitions were observed[54]. Reprinted with permission from Jiang et al.[54]. Copyright 2015 by American Physical Society.

$\mathrm{CaFeAs}_{2}[28], \mathrm{Ca}_{1-\mathrm{x}} \mathrm{La}_{\mathbf{x}} \mathrm{FeAs}_{2}(x=0.27)[54,74]$ is the non-superconducting parent compound of this family which is naturally structurally detwinned at ambient pressure and becomes superconducting on electron or hole-doping. Neutron diffraction and muon spin rotation $(\mu \mathrm{SR})$ measurements suggested a structural phase transition from a monoclinic to a triclinic phase at $58 \mathrm{~K}$ and a paramagnetic to stripe AFM phase transition at $54 \mathrm{~K}$ (Fig. 2), both of which can be suppressed by Cosubstitution on the Fe-sites[54]. Additionally, the presence of two different crystallographic phases in different temperature regions were further confirmed from an optical $2^{\text {nd }}$ harmonic generation study[74] where no significant modification of the electronic structure was observed as a result of the phase transitions. A similar structural phase transition was recently reported in superconducting $\mathrm{Ca}_{1-\mathrm{x}} \mathrm{La}_{\mathrm{x}} \mathrm{FeAs}_{2}(x=0.15)$ around $100 \mathrm{~K}$ using temperature dependent X-ray measurements[77] while Kawasaki et al.[51] reported an AFM ordering around $T_{\mathrm{N}} \sim 62 \mathrm{~K}$ for a sample with bulk $T_{\mathrm{c}} \sim 35 \mathrm{~K}$. This suggests a correlation between the structural and AFM phase transition in $\mathrm{Ca}_{1-\mathrm{x}} \mathrm{La}_{\mathrm{x}} \mathrm{FeAs}_{2}$ occurring in similar 


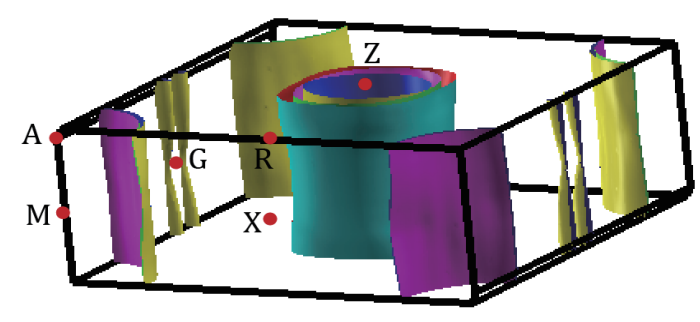

FIG. 3: DFT calculated Fermi surface of $\mathrm{CaFeAs}_{2}$ in the nonmagnetic state[53]. Reprinted with permission from Huang et al. [53]. Copyright 2015 by American Institute of Physics.

temperature window and a weak coupling between the structural and magnetic order.

Upon Co-doping into $\mathrm{Ca}_{1-\mathrm{x}} \mathrm{La}_{\mathrm{x}} \mathrm{FeAs}_{2}(x=0.2)$ [49], a mixture of 112- and 122-phases were observed. For low Co-content, almost pure 112-phase was found with monoclinic structure, but with an enhancement of the Co-doping level a mixture of the 112 and 122-phases were observed which for a higher Co-doping $(>6 \%)$ resulted even in the complete disappearance of the 112phase. Owing to the slightly smaller ionic radius of $\mathrm{Co}^{2+}$ (74 pm) compared to $\mathrm{Fe}^{2+}(76 \mathrm{pm})$, the successful substitution could be confirmed from the shift of the out-ofplane X-ray reflection. This suggests that the structural stability of the 112-phase only exists in a narrow window of Co-doping level putting strong constraints to the crystal growth conditions.

\section{Electronic Structure}

\section{A. Theoretical Investigations}

Density functional theory (DFT) band structure analysis of fully stoichiometric $\mathrm{LaPdBi}_{2}$ revealed the almost equal contribution of all constituent atoms near the Fermi level, except for $\mathrm{Pd}$ which has a much higher dominance. Changing the $\mathrm{Pd}$ content in $\mathrm{CePd}_{\mathrm{x}} \mathrm{Bi}_{2}$ affects the Fermi surface topology significantly and in the presence of significant $\mathrm{Pd}$ vacancies, Fermi surface nesting can be avoided. This suppresses any kind of charge density wave (CDW) in the Bi square net layer which allows the superconducting state to stabilise[56].

The density of states of two hypothetical 112structures $\mathrm{BaFeAs}_{2}$ and $\mathrm{BaFeSb}_{2}[28]$ showed a considerable amount of $\mathrm{Fe}-3 d$ states at $E_{\mathrm{F}}$ with a small contribution from the spacer layer. This has been claimed to originate from the larger distance of separation between the $\mathrm{As}(1)$ and FeAs layers with minimal hybridisation between them. For $\mathrm{CaFeAs}_{2}$, first-principles calculation also suggested the strong presence of Fe-3d electrons in the density of states near the Fermi level [16, 53]. The Fermi surface of $\mathrm{CaFeAs}_{2}$ consists of two electron cylinders at the Brillouin zone (BZ) corner ( $M$ point), four Dirac cone type electron cones at the BZ edge $(G$ point) and three hole cylinders with an additional

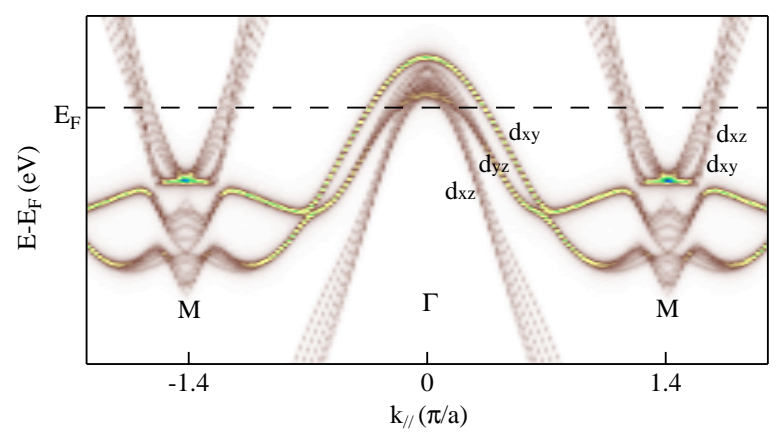

FIG. 4: Band structure of $\mathrm{Ca}_{1-\mathrm{x}} \mathrm{La}_{\mathrm{x}} \mathrm{FeAs}_{2}$ projected to the in-plane BZ as obtained from ARPES measurements[52]. Reprinted with permission from Liu et al.[52]. Copyright 2015 by American Institute of Physics.

three-dimensional (3D) hole pocket at the BZ centre $(\Gamma$ point) $[16,53,78,79]$ as illustrated in Fig. 3. The presence of the additional hole pocket at $(0,0,0)$ (likely originating from the hybridisation between the Fe $3 d_{x z} / 3 d_{y z}$ and $\operatorname{As}(1) 4 p$ orbitals from the FeAs layer) and four electron cones at the $G$ point (contributed by $\operatorname{As}(2) p$-states) have not been found earlier in $1111[80,81]$ and $122[82]-$ pnictide phases. The nesting between the $\Gamma$ point hole pockets and $G$ point electron cones possibly results into a AFM spin density wave (SDW) phase which gets suppressed upon $R E$-doping favoring the superconducting state[53, 83].

The band dispersion in $\mathrm{Ca}_{1-\mathrm{x}} \mathrm{La}_{\mathrm{x}} \mathrm{FeAs}_{2}$ showed considerable $2 \mathrm{D}$ character contributed mostly by the As layers. In the non-magnetic calculation, four hole-like bands (around $\Gamma(0,0)$ point) and two electron-like bands (around $M(\pi, \pi)$ point) were found from the band structure analysis (see Fig. 4) which has some similarity with $\mathrm{BaFe}_{2} \mathrm{As}_{2}$ [78]. It was predicted that for $T<T_{\mathrm{c}}$, $\mathrm{Ca}_{1-\mathrm{x}} \mathrm{La}_{\mathrm{x}} \mathrm{FeAs}_{2}$ could work as a natural topological insulator/superconductor hybrid structure (FeAs layer to be responsible for the superconductivity and the As chain layer being the topological insulator) that could be ideal for the realisation of Majorana fermions[84].

Nagai et al. [85] investigated the effect of Sbsubstitution on the superconducting properties of $\mathrm{CaFe}\left(\mathrm{Sb}_{x} \mathrm{As}_{1-x}\right)_{2}$. Sb-doping in the As zig-zag layer increased the lattice parameters $a, b$ with an overall increase in the unit cell volume leading to an overall stabilisation of the structure which is energetically more favourable than substitution in the FeAs layer. However, the calculated band structure with and without Sbdoping is very similar except a small shift along the $G-\Gamma$ direction. Primary investigations suggested the role of Sb-substitution in the enhancement of $T_{\mathrm{c}}$ to be possibly related to the stabilisation of the As-chains which have a crucial role in controlling $T_{\mathrm{c}}$. 


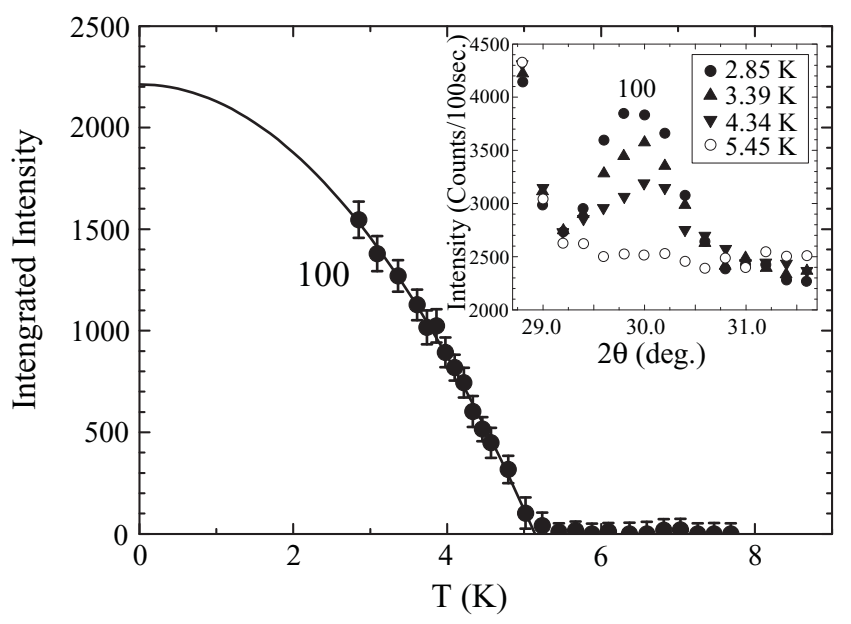

FIG. 5: Temperature dependence of the integrated intensity of the (100) Bragg peak as obtained from neutron diffraction measurements of $\mathrm{CeNi}_{\mathrm{x}} \mathrm{Bi}_{2}$. The solid line describes a $\left(1-\left(T / T_{N}\right)^{2}\right)$ dependence of the intensity below $5 \mathrm{~K}[36]$. Reprinted with permission from Kodama et al.[36]. Copyright 2011 by American Physical Society.

\section{B. Experimental Results}

ARPES measurements on $\mathrm{Ca}_{1-x} \mathrm{La}_{\mathrm{x}} \mathrm{FeAs}_{2}$ indicated a band structure similar to the other pnictide systems consisting of three hole like bands $\left(d_{x z}, d_{y z}, d_{x y}\right.$ character) at the $\Gamma$ point and two electron like bands $\left(d_{x z}, d_{x y}\right.$ types) at the $M$ point of the BZ[50, 52, 54, 78], with reasonable nesting mostly originating from the $\mathrm{Fe}-3 d$ electrons (see Fig. 4). No evidence of the As- $p$ or Ca$d$ states were found in the Fermi surface map scanned over a complete 3D momentum space which is similar to the absence of interstitial blocks on the Fermi surface in 1038-phase (CaFeAs) ${ }_{10} \mathrm{Pt}_{3.58} \mathrm{As}_{8}$ and 1048phase $\left(\mathrm{CaFe}_{0.95} \mathrm{Pt}_{0.05} \mathrm{As}\right)_{10} \mathrm{Pt}_{3} \mathrm{As}_{8}[86]$ and 1111-phase $\mathrm{SmFe}_{1-x} \mathrm{Co}_{x} \mathrm{AsO}[87]$ systems, but in contrast to the predictions made from electronic structure calculations [83]. However, Li et al.[50] managed to resolve the presence of an additional 3D hole like band at the zone centre and a fast disappearing band near the $\mathrm{X}$-point at the zone corner in $\mathrm{Ca}_{1-\mathrm{x}} \mathrm{La}_{\mathrm{x}} \mathrm{FeAs}_{2}(\mathrm{x}=0.1)$ and also for $x=0.27[54]$. The presence of a considerable As- $4 p_{z}$ orbital for the hole like band and As- $4 p_{x, y}$ orbitals for the narrow band were resolved while considerable hybridisation between the As$4 p_{z}$ and Fe-3d orbitals were found in the hole-like band.

\section{Magnetic Structure}

The layered compound $\mathrm{CePd}_{\mathrm{x}} \mathrm{Bi}_{2}$ is a Kondo-lattice AFM which is metallic above $75 \mathrm{~K}$, and exhibits below $75 \mathrm{~K}$ a strong interplay between Kondo and crystal field effects (CEF)[73]. Magnetic susceptibility data suggested that the material is anisotropic due to the CEF effects with an AFM ordering temperature $T_{\mathrm{N}}=6 \mathrm{~K}[56]$. The high-temperature susceptibility fitted using the Curie-

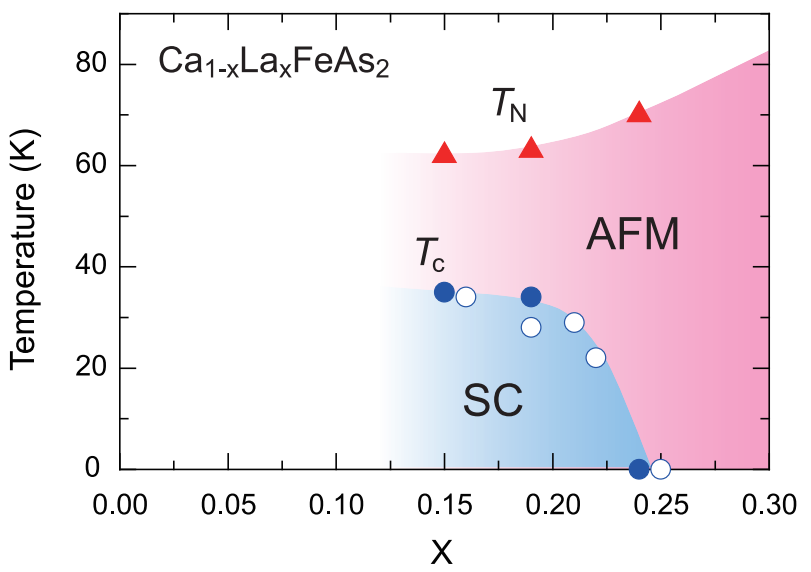

FIG. 6: Phase diagram of $\mathrm{Ca}_{1-\mathrm{x}} \mathrm{La}_{\mathrm{x}} \mathrm{FeAs}_{2}$ for various doping levels of La[51]. Reprinted with permission from Kawasaki et al.[51]. Copyright 2015 by American Physical Society.

Weiss law $\chi=C /\left(T-\theta_{c}\right)$ revealed a Curie Temperature $\theta_{c}=-1.5 \mathrm{~K}$ and an effective magnetic moment $\mu_{\mathrm{eff}}=2.86 \mu_{B} /$ Ce atom which is of similar value as for a free $\mathrm{Ce}^{3+}$ ion indicating the localised nature of the Cemoments in $\mathrm{CePd}_{\mathrm{x}} \mathrm{Bi}_{2}$. The negative $\theta_{c}$ supports the existence of AFM ordering of the Ce-atoms at higher temperatures[56]. The Hall-coefficient $R_{\mathrm{H}}$ of $\mathrm{CePd}_{\mathrm{x}} \mathrm{Bi}_{2}$ is negative with an average value of $1.7 \times 10^{-4} \mathrm{~cm}^{3} / \mathrm{C}$ which corresponds to a carrier concentration of $3.7 \times 10^{22} / \mathrm{cm}^{3}$. $R_{\mathrm{H}}$ stays temperature independent suggesting a singleband character of the carriers [56]. The strong interplay between the Kondo and CEF interactions leads to a reconstruction of the Fermi surface topology which is most likely responsible for the absence of superconductivity in $\mathrm{CePd}_{\mathrm{x}} \mathrm{Bi}_{2}$ as iso-structural $\mathrm{LaPd}_{\mathrm{x}} \mathrm{Bi}_{2}$ is superconducting and both have similar Fermi surfaces above $75 \mathrm{~K}$. Electronic structure calculation suggested that the $\mathrm{Pd}$ vacancies induce strong scattering effects in the $\mathrm{Pd}_{x} \mathrm{Bi}$ layer that enhances the CEF effect to quench the Ce moments at low temperature. The Fermi surface reconstruction and Ruderman-Kittel-Kasuya-Yoshida (RKKY) interaction induced magnetic ordering can induce superconductivity in the heavy-fermion system $\mathrm{CeCu}_{2} \mathrm{Si}_{2}$ [88], which is unlikely to occour in the case of $\mathrm{CePd}_{\mathrm{x}} \mathrm{Bi}_{2}$ without external influences[73].

The ground state of the Ce-based intermetallic compound $\mathrm{CeNi}_{x} \mathrm{Bi}_{2}$ is governed by the interplay between RKKY and Kondo interactions. The Kondo interaction strength is determined by the level of hybridisation between the Ce- $4 f$ and conduction electrons favoring a nonmagnetic ground state while the long range magnetically ordered state is preferred by the RKKY interaction[39]. The parent compound of $\mathrm{CeNi}_{\mathrm{x}} \mathrm{Bi}_{2}$ is $\mathrm{CeNiBi}_{2}$ which is a moderately heavy-fermion antiferromagnet with a magnetic ordering temperature $T_{\mathrm{N}}$ of $5 \mathrm{~K}[36]$. Rosa et al.[32] reported that $T_{\mathrm{N}}$ in $\mathrm{CeNi}_{x} \mathrm{Bi}_{2-y}$ increases with an increase in $x$, which also enhances the magnetic anisotropy of $\mathrm{Ce}^{3+}$ at low temperature. Below $5 \mathrm{~K}$, the Ce moments 


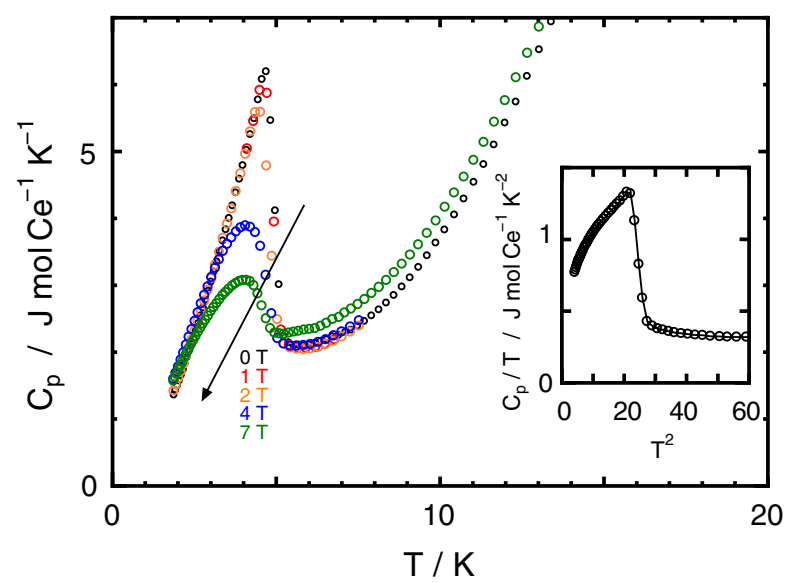

FIG. 7: Temperature dependence of the specific heat $\left(C_{p}\right)$ for different applied magnetic fields with distinct $\lambda$ peak around $5 \mathrm{~K}$ in $\mathrm{CeNi}_{\mathrm{x}} \mathrm{Bi}_{2}$. Inset: $C_{p} / T-T^{2}$ at $0 \mathrm{Oe}[33]$. Reprinted with permission from Mizoguchi et al.[33]. Copyright 2011 by American Physical Society.

order antiferromagnetically with the easy magnetisation axis along the $c$-direction with a saturated magnetic moment of $1.71 \mu_{B}$ as $T \rightarrow 0 \mathrm{~K}$. Superconductivity is induced in $\mathrm{CeNiBi}_{2}$ via Ni-deficiency similar to the oxygen deficiency in the 1111-system[89]. From powder neutron diffraction measurements (see Fig. 5) clear Bragg peaks are observed at $q=(0,0,0)$ below the magnetic ordering temperature $T_{\mathrm{N}} \sim 5.45 \mathrm{~K}$ and the intensity of the (100) Bragg peak increases down to the lowest temperature. However, no anomaly or jump was observed in the peak intensity around $T_{\mathrm{c}}$ which is unlike the features observed in heavy-fermion superconductors where Bragg intensities are suppressed below $T_{\mathrm{c}}[90]$. This suggested that Ce- $4 f$ electrons do not contribute to the superconductivity and the material cannot be considered to be a heavy-fermion superconductor. Although $\mathrm{CeNi}_{\mathrm{x}} \mathrm{Bi}_{2}$ has a similar crystal structure like 1111-pnictide, the $T_{\mathrm{c}}$ is much lower than in the 1111-system which can be due to the absence of magnetic fluctuations in $\mathrm{CeNi}_{\mathbf{x}} \mathrm{Bi}_{2}[36]$. Anisotropic magnetic behavior was observed in the single crystalline $R E \mathrm{Ni}_{x} \mathrm{Bi}_{2 \pm y}$ compounds $(R E=\mathrm{Ce}-\mathrm{Nd}, \mathrm{Sm}$, Gd-Dy) which order antiferromagnetically at low temperatures between $3.3 \mathrm{~K}(\mathrm{Sm})$ and $10.2 \mathrm{~K}(\mathrm{~Tb})[39]$.

From first-principles based investigation, the ground state of $\mathrm{CaFeAs}_{2}$ was predicted to be a SDW type striped AFM phase driven by Fermi surface nesting with the magnetic moment of each Fe atom to be $2.1 \mu_{B}$ [53], significantly smaller in value than the LDA calculated value for 1111-LaFeAsO [16] and the hypothetical 112compounds $\mathrm{BaFeAs}_{2}$ and $\mathrm{BaFeSb}_{2}[28]$. Electron doping using rare-earth elements can help suppressing the SDW state and stabilising the superconducting state. Nuclear magnetic resonance (NMR) measurements revealed that the AFM ordering sets in below $T_{\mathrm{N}}=62 \mathrm{~K}$ for highly doped $\mathrm{Ca}_{1-\mathrm{x}} \mathrm{La}_{\mathrm{x}} \mathrm{FeAs}_{2}(x=0.15)$ while superconductiv- ity sets in at $T_{\mathrm{c}}=35 \mathrm{~K}[51]$. With an increase in the doping concentration, AFM order gets enhanced as $T_{\mathrm{N}}$ rising up to $70 \mathrm{~K}[51]$ for $x=0.24$ (see Fig. 6) which possibly originates from the nesting of the Fermi surfaces due to additional electron doping by $\mathrm{La}[50,78]$. The AFM order in $\mathrm{Ca}_{1-\mathrm{x}} \mathrm{La}_{\mathrm{x}} \mathrm{FeAs}_{2}$ has been found to be robust against $R E$ doping. A similar phase diagram was also obtained for the 1111-system $\mathrm{LaFeAsO}_{1-x} \mathrm{H}_{x}[91,92]$ where heavy doping enhanced $T_{\mathrm{N}}$ and, in both cases, a structural phase transition was observed above $T_{\mathrm{N}}[54,92]$. However, the superconducting and AFM orders coexist in $\mathrm{Ca}_{1-\mathrm{x}} \mathrm{La}_{\mathrm{x}} \mathrm{FeAs}_{2}$ microscopically[51], while both orders are completely segregated in $\mathrm{LaFeAsO}_{1-x} \mathrm{H}_{x}$ [93].

\section{Superconducting Properties}

\section{A. Low Critical Temperature Materials}

$\mathrm{CeNi}_{\mathrm{x}} \mathrm{Bi}_{2}$ is a type-II superconductor $\left(T_{\mathrm{c}}=4.2 \mathrm{~K}\right)[33]$ with a SVF of $96 \%$. Below $10 \mathrm{~K}$, the resistivity shows a $\rho(T) \sim T^{2}$ dependence and increases linearly with temperature up to $100 \mathrm{~K}$. For other $R E$-containing $R E \mathrm{Ni}_{x} \mathrm{Bi}_{2}$ systems with $R E=\mathrm{La}, \mathrm{Nd}, \mathrm{Y}$, also $T_{\mathrm{c}}$ values around $4 \mathrm{~K}$ were measured, but the SVF was significantly less than the Ce-containing phase. The specific heat data for $\mathrm{CeNi}_{\mathrm{x}} \mathrm{Bi}_{2}$ (see Fig. 7) suggested the presence of two different types of carries with different effective masses. The AFM ordering around $5 \mathrm{~K}$ was caused by the heavy carriers originating from the magnetic interaction between conduction electrons and Ce- $4 f$ electrons in the $\mathrm{Ni}_{x} \mathrm{Bi}$ plane, while the light carriers from the $\mathrm{Bi}$ square net layer were responsible for superconductivity. The entropy calculation suggested that the sharp jump in the specific heat around $T_{\mathrm{c}}$ corresponds to the magnetic ordering of Ce- $4 f$ moments, which are not involved in the occurrence of superconductivity. Lin et al.[39] claimed that the observed superconducting properties of $\mathrm{LaNi}_{\mathrm{x}} \mathrm{Bi}_{2}$ and $\mathrm{CeNi}_{\mathrm{x}} \mathrm{Bi}_{2}$ are most likely due to the presence of $\mathrm{Bi}$ and $\mathrm{Ni}-\mathrm{Bi}$ binary impurities as very little evidence of bulk superconductivity was found from their single crystalline samples. However, highly crystalline thin films also showed superconductivity around $4 \mathrm{~K}[34,55]$. The zero-resistance state in $\mathrm{LaPd}_{x} \mathrm{Bi}_{2}$ [56] was observed around $2 \mathrm{~K}$ with a bulk superconducting phase around the same temperature which is likely $s$-wave in nature. In the normal state, it is metallic with a residual resistivity ratio (RRR) of 2.45 indicating strong scattering in the conducting layers.

\section{B. High Critical Temperature Materials}

\section{Rare Earth Substitution}

The predicted mother compound of the $(\mathrm{Ca}, R E)-112$ family $\mathrm{CaFeAs}_{2}$ has not been synthesised yet and superconductivity was only observed in the $R E$-doped systems within a limited doping range. Doping of $R E$-elements also improved the $T_{\mathrm{c}}$ value of 122-type (Ca, RE) $\mathrm{Fe}_{2} \mathrm{As}_{2}$ with $T_{\mathrm{c}}^{\text {onset }}$ values : $\mathrm{Ca}_{1-x} \mathrm{Pr}_{x} \mathrm{Fe}_{2} \mathrm{As}_{2} \quad(47 \mathrm{~K})[94]$, 

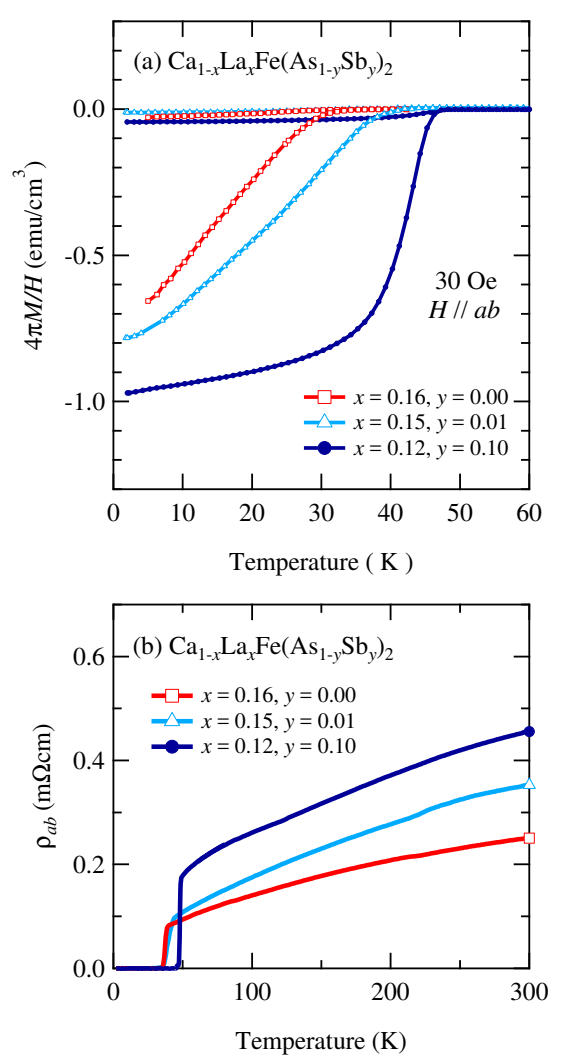

FIG. 8: Temperature dependence of the (a) magnetisation at $H(\| a b)=30 \mathrm{G}$ in $\mathrm{ZFC}$ and $\mathrm{FC}$ conditions and (b) inplane resistivity $(\rho \| a b)$ for different La and Sb-doping in $\mathrm{Ca}_{1-x} \mathrm{La}_{x} \mathrm{Fe}\left(\mathrm{As}_{1-y} \mathrm{Sb}_{y}\right)_{2}[18]$. Reprinted with permission from Kudo et al.[18]. Copyright 2014 by Physics Society of Japan.

$\mathrm{Ca}_{1-x} \mathrm{La}_{x} \mathrm{Fe}_{2}\left(\mathrm{As}_{1-y} \mathrm{P}_{y}\right)_{2} \quad$ (45K)[95], $\mathrm{Ca}_{1-x} \mathrm{Pr}_{x} \mathrm{Fe}_{2} \mathrm{As}_{2}$ $(49 \mathrm{~K})[96]$. However, complete appearance of bulk superconductivity was not observed in some of these cases, possibly relating to the filamentary nature of the superconducting phase.

The in-plane resistivity of $\mathrm{Ca}_{1-\mathrm{x}} \mathrm{La}_{\mathrm{x}} \mathrm{FeAs}_{2}$ with $x=$ 0.16 goes through a superconducting transition at $36 \mathrm{~K}$ [16] with a transition width of $2.4 \mathrm{~K}$ and bulk superconductivity was observed from magnetisation measurements at $34 \mathrm{~K}$ with a SVF of $66 \%$. For $x=0.21$, $T_{c}^{\text {onset }}$ increased to $45 \mathrm{~K}$ [16], but the zero-resistance state was only observed at $25 \mathrm{~K}$ which is consistent with the $T_{\mathrm{c}}$ determined from the magnetisation data. In $\left(\mathrm{Ca}_{0.9} \mathrm{Pr}_{0.1}\right) \mathrm{Fe}_{1.3} \mathrm{As}_{1.8} \mathrm{O}_{0.2}$, a small level of O-doping helped enhancing the SVF as bulk superconductivity was observed with a $T_{\mathrm{c}}$ of $20 \mathrm{~K}[40]$.

Replacing As by a small amount of isovalent substitutional elements like $\mathrm{P}$ and $\mathrm{Sb}$ enhanced $T_{\mathrm{c}}$ further [17]. For $5 \% \mathrm{P}$ doping in $\mathrm{Ca}_{1-x} \mathrm{La}_{x} \mathrm{Fe}\left(\mathrm{As}_{0.995} \mathrm{P}_{0.005}\right)_{2}$ $(x=0.16)$ bulk $T_{\mathrm{c}}$ was enhanced to $41 \mathrm{~K}$ with a SVF of $44 \%$ at $5 \mathrm{~K}$. For $x=0.18, T_{\mathrm{c}}$ got reduced to
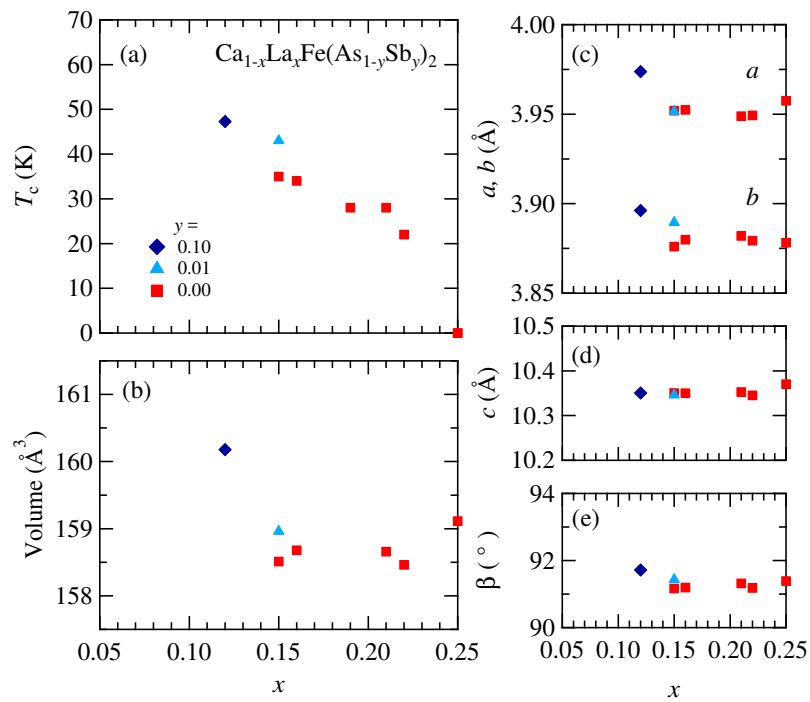

FIG. 9: Dependence of (a) $T_{\mathrm{c}}$, (b) unit cell volume, (c) $a$, $b$ lattice constants, (d) $c$-lattice constant, and (e) $\beta$ angle of $\mathrm{Ca}_{1-x} \mathrm{La}_{x} \mathrm{Fe}\left(\mathrm{As}_{1-y} \mathrm{Sb}_{y}\right)_{2}$ for various La-doping levels $x$.[18]. Reprinted with permission from Kudo et al.[18]. Copyright 2014 by Physics Society of Japan.

$39 \mathrm{~K}$, but the SVF increased to $77 \%$. For Sb-doped $\mathrm{Ca}_{1-x} \mathrm{La}_{x} \mathrm{Fe}\left(\mathrm{As}_{0.99} \mathrm{Sb}_{0.01}\right)_{2}(x=0.16)$, the bulk $T_{\mathrm{c}}$ increased to $43 \mathrm{~K}$ with a significant SVF of $78 \%$. From the trend in the $T_{\mathrm{c}}$ vs. $x$ phase diagram in Fig. 9(a), the highest $T_{\mathrm{c}}$ was found for all materials at the lowest $x$ value. This suggests that a much higher $T_{\mathrm{c}}$ could be expected if $x$ can be reduced further below 0.15 which needs development of chemical synthesis techniques.

Further enhancement of $T_{\mathrm{c}}$ was possible with a higher level of Sb substitution in $\mathrm{Ca}_{1-x} \mathrm{La}_{x} \mathrm{Fe}\left(\mathrm{As}_{1-y} \mathrm{Sb}_{y}\right)_{2}(x=$ $0.12, y=0.1$ ) to $47 \mathrm{~K}[18]$ with a SVF of $100 \%$ at $2 \mathrm{~K}$ (see Fig. 8) indicating the appearance of complete bulk superconductivity. This enhanced $T_{\mathrm{c}}$ could originate from two different effects: (1) simultaneous Sb doping, (2) decrease in La content resulting in an increase in cell volume (Fig. 9(b)). The decrease in La content suggests the reduction of the number of charge carriers as the ionic radii of $\mathrm{La}^{3+}$ and $\mathrm{Ca}^{2+}$ are comparable. Secondly, additional $\mathrm{Sb}$ substitution can induce a negative chemical pressure due to an increase in cell volume as the ionic radius of $\mathrm{Sb}^{3-}\left(\mathrm{Sb}^{-}\right)$is larger than $\mathrm{As}^{3-}\left(\mathrm{As}^{-}\right)$which was observed from the increase in the $a, b$ lattice constants (see Fig. 9(c-d)) and the higher level of localisation of the $d$-electrons with a larger sized $P n$ atom. However, this mechanism is not valid for the $T_{\mathrm{c}}$ enhancement in the P-doped system[17] as neither the La content got reduced nor the lattice parameters changed in that case.

In Sb-substituted $\mathrm{Ca}_{1-x} R E_{x} \mathrm{Fe}\left(\mathrm{As}_{1-y} \mathrm{Sb}_{y}\right)_{2}$, there is a general trend of $T_{\mathrm{c}}$ enhancement and improvement of the superconducting properties[18]. In the absence of $\mathrm{Sb}(y=0)$, for the Ce-doped system, no evidence of bulk superconductivity was observed, while $\mathrm{Pr}$ - and Nd-doped 

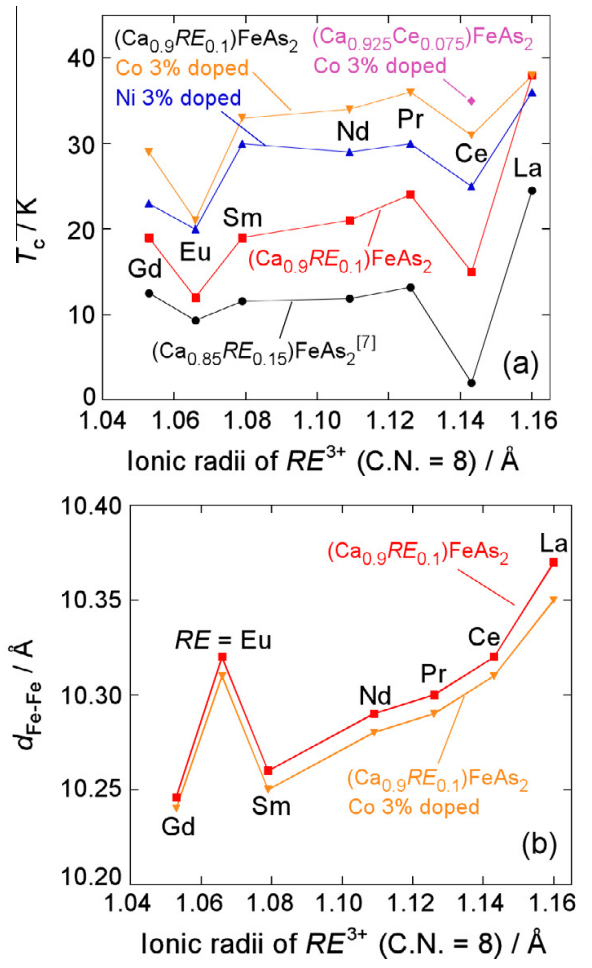

FIG. 10: (a) $T_{\mathrm{c}}$ and (b) $d_{F e-F e}$ of $(\mathrm{Ca}, R E) \mathrm{FeAs}_{2}$ as function of the ionic radii of various $R E^{3+}$ ions with coordination number eight[42]. Reprinted with permission from Yakita et al.[42]. Copyright 2015 by Elsevier.

systems exhibited $T_{\mathrm{c}}$ at $10 \mathrm{~K}$ and $11 \mathrm{~K}$ respectively with a SVF of $5 \%$. For the Ce-doped system, the origin of the zero-resistance state was attributed to filamentary superconductivity as no clear diamagnetic signal was observed at $T_{\mathrm{c}}$. Sb-doping improved superconductivity with a $T_{\mathrm{c}}$ of $21 \mathrm{~K}(y=0.01)$ and $43 \mathrm{~K}(y=0.1)$ for the Ce-doped system, $26 \mathrm{~K}(y=0.01)$ and $43 \mathrm{~K}(y=0.05)$ for the Prdoped system, $24 \mathrm{~K}(y=0.01)$ and $43 \mathrm{~K}(y=0.05)$ for the Nd-doped systems with a substantial increase in the SVF indicating the appearance of bulk superconductivity in all these phases. This suggests that irrespective of the $R E$ element, a $T_{\mathrm{c}}$ above $40 \mathrm{~K}$ can be obtained by tuning the $\mathrm{Sb}$ content. Due to Sb substitution, the $b$-lattice parameter increased which helped enhancing $T_{\mathrm{c}}$, but the $c$-value stayed almost unchanged for $x=0.15-0.25$ (see Fig. 9(c-e)). Kudo et al. [18] suggested that $T_{\mathrm{c}}$ can be increased beyond $50 \mathrm{~K}$ if the $b$-lattice constant becomes equal to $a$ with a simultaneous reduction of the $c$-value to adjust the As-Fe-As bond angle.

In polycrystalline $\mathrm{Ca}_{1-x} R E_{x} \mathrm{FeAs}_{2}$, superconductivity was observed with a $T_{\mathrm{c}}$ of $22.7 \mathrm{~K}, 24.6 \mathrm{~K}, 17.9 \mathrm{~K}$, $23.2 \mathrm{~K}, 13.2 \mathrm{~K}$ and $22.8 \mathrm{~K}$ for La-, Pr-, Nd-, Sm-, Euand $\mathrm{Gd}$-doped systems respectively, except for the Cedoped system[48]. Broad resistive transitions were observed due to the inhomogeneous distribution of the $R E$ atoms resulting in poor grain connectivity in the polycrystalline phases. There is an indication of a decrease

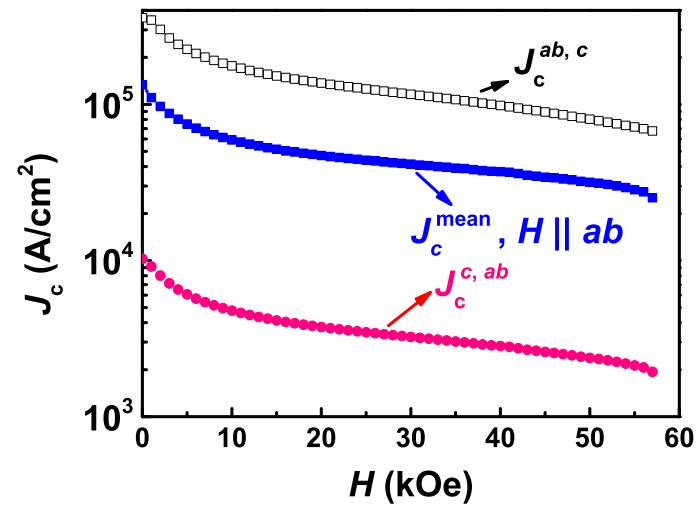

FIG. 11: Magnetic field dependence of critical current density $J_{c}$ of $\mathrm{Ca}_{1-\mathrm{x}} \mathrm{La}_{\mathrm{x}} \mathrm{FeAs}_{2}$ in the in-plane $(H \| a b)$ and out-ofplane $(H \| c)$ configuration[47]. Reprinted with permission from Zhou et al.[47]. Copyright 2014 by Japanese Society of Applied Physics.

of $T_{\mathrm{c}}$ with decrease of the ionic radii of the $R E$ atoms, a similar behavior was observed for $d_{F e-F e}$ (interlayer distance between the neighbouring Fe planes). Note that none of these trends are clearly established due to the inhomogeneity of the phases. Compositional analysis revealed that the actual level of Ce in the Ca site is high due to the similar ionic radii [42] which could be a reason behind the absence of superconductivity in the Ce-doped system.

\section{Transition Metal Substitution}

In the $R E$-substituted material $\mathrm{Ca}_{1-x} R E_{x} \mathrm{FeAs}_{2}$, transition metal $(T M)$-co-doping indicates the direct substitution of the Fe atoms by Co-atoms which has been studied for $T M=\mathrm{Co}, \mathrm{Ni}, \mathrm{Mn}[42,49,72]$. Mn-codoping suppressed superconductivity completely. However, it was found that a small level $(3 \%)$ of Coand Ni-doping improved the superconducting properties of the $(\mathrm{Ca}, R E) 112$-systems[42, 49]. Co-co-doped $\mathrm{Ca}_{1-x} R E_{x}\left(\mathrm{Fe}_{1-y} \mathrm{Co}_{y}\right) \mathrm{As}_{2}$ has an enhanced $T_{\mathrm{c}}$ above $30 \mathrm{~K}$ for $R E=\mathrm{La}, \mathrm{Ce}, \mathrm{Pr}, \mathrm{Nd}, \mathrm{Sm}$, in spite of the direct $T M$ substitution on the Fe-sites. This effect is similar for Ni-doped systems with slightly lower $T_{\mathrm{c}}$ compared to the Co-doped phases suggesting an overdoped state of the Ni-doped systems. On 3\% Co-co-doping, the $T_{\mathrm{c}}$ of the $(\mathrm{Ca}, \mathrm{Pr})-112$ system increased significantly from $23 \mathrm{~K}$ (TM-free case) to $36 \mathrm{~K}[72]$, while also the superconducting transition became sharper suggesting improved grain connectivity with significant enhancement in the $T_{c}^{\text {zero }}$ value (from $14 \mathrm{~K} \rightarrow 30 \mathrm{~K}$ ) and no change in the $T_{\mathrm{c}}$ onset value. With an increase in the Co-doping level, a linear suppression of $T_{\mathrm{c}}{ }^{\text {zero }}$ and $T_{\mathrm{c}}{ }^{\text {onset }}$ was observed at an average rate of $1.65 \mathrm{~K} / \mathrm{Co} \%$ [49].

Large diamagnetic screening was observed in the Coco-doped (Ca, La)-112-system indicating the presence of bulk superconductivity[42], although it was not clear why a direct substitution of Co for Fe would result in 


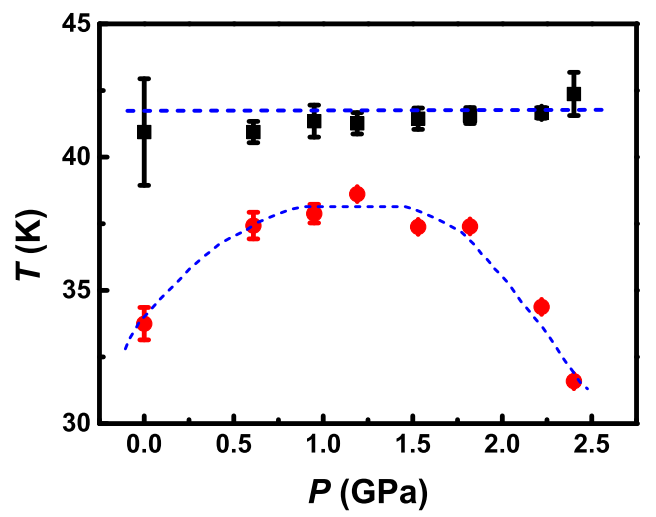

FIG. 12: Pressure dependence of $T_{1}$ and $T_{\mathrm{c}}{ }^{\text {zero }}$ of $\mathrm{Ca}_{1-\mathrm{x}} \mathrm{La}_{\mathrm{x}} \mathrm{FeAs}_{2}$ with $x=0.18$ single crystals[45]. Reprinted with permission from Zhou et al.[45]. Copyright 2015 by Institute of Physics.

such behavior which is also contrary to doping effects in 122- and 1111-phases [97, 98]. However, the improved superconducting properties could arise from the decrease in La-content in the Co-doped phases, similar to Sb doping in (Ca,La)-112[18] which resulted in an optimisation of the As-Fe-As bond angle.

In $\mathrm{Ca}_{1-x} R E_{x}\left(\mathrm{Fe}_{1-y} T M_{y}\right) \mathrm{As}_{2}(T M=\mathrm{Co}, \mathrm{Ni}), T_{\mathrm{c}}$ increased with increasing ionic radii for all $R E$ elements except for $\mathrm{Ce}$ and $\mathrm{Eu} . \quad d_{\mathrm{Fe}-\mathrm{Fe}}$ went through a similar trend with ionic radii which is comparable to the trend observed earlier in the $T M$-free (Ca,RE)112-system[48] (see Fig. 10). Co-co-doping resulted in slight reduction of $d_{\mathrm{Fe}-\mathrm{Fe}}$ for all $(\mathrm{Ca}, \mathrm{RE}) 112$-systems, keeping the $d_{\mathrm{Fe}-\mathrm{Fe}}$ vs ionic radii trend the same for $\mathrm{Co}$-free and Co-doped cases. For the (Ca,Ce)112-system, the reduction of the Ce-content increased the $T_{\mathrm{c}}$ and with Co-co-doping followed the same trend. For the Eu-doped system, an exceptionally low $T_{\mathrm{c}}$ and large $d_{\mathrm{Fe}-\mathrm{Fe}}$ was measured which most likely indicates the coexistence of $\mathrm{Eu}^{2+}$ and $\mathrm{Eu}^{3+}$.

\section{Critical Current and Critical Field}

The lower critical fields $\left(H_{c 1}\right)$ for $\mathrm{CeNi}_{\mathrm{x}} \mathrm{Bi}_{2}, \mathrm{LaNi}_{x} \mathrm{Bi}_{2}$, $\mathrm{NdNi}_{x} \mathrm{Bi}_{2}, \mathrm{YNi}_{x} \mathrm{Bi}_{2}$ are $65 \mathrm{G}, 90 \mathrm{G}, 55 \mathrm{G}$ and $67 \mathrm{G}$ respectively [33]. The upper critical fields $\left(H_{c 2}\right)$ at zerotemperature for $\mathrm{LaPd}_{x} \mathrm{Bi}_{2}$ was $3 \mathrm{~T}$ [56] which is relatively large for a $T_{\mathrm{c}} \sim 2 \mathrm{~K}$ suggesting type-II superconductivity. For $\mathrm{Ca}_{1-\mathrm{x}} \mathrm{La}_{\mathrm{x}} \mathrm{FeAs}_{2}$, critical fields near $T=0$ are as high as $H_{c 2}^{c}(0)=39.4 \mathrm{~T}$ (for $H \| c$ ) and $H_{c 2}^{a b}(0)=$ $166.2 \mathrm{~T}$ (for $H \| a b$ ) which corresponds to coherence lengths $[47,77]$ of $\xi_{c}(0)=6.9 \AA$ and $\xi_{a b}(0)=28.9 \AA$. The anisotropy $\gamma(0)$ near $T_{\mathrm{c}}$ is 2.8 which lies in between $1111(5<\gamma<9.2)[99]$ - and $122(1<\gamma<2)[100]-$ type pnictides. $\gamma$ is a measure of the interlayer coupling strength between the FeAs and charge reservoir layers suggesting the presence of moderate anisotropy in 112-system. The anisotropic pinning potential in $\mathrm{Ca}_{1-\mathrm{x}} \mathrm{La}_{\mathrm{x}} \mathrm{FeAs}_{2}$ showed a field dependence for $H \| a b$ similar to the cuprates and the 1111-system[99] suggest-
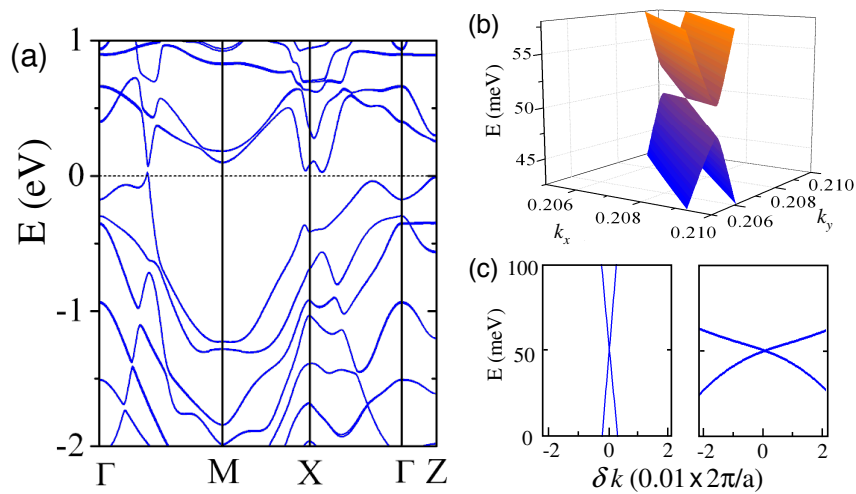

FIG. 13: (a) Electronic band structure of $\mathrm{SrMnBi}_{2}$. (b) Anisotropic energy surfaces around the Dirac point $k_{0}=$ $(0.208,0.208)$ for the $(\mathrm{SrBi})^{+}$layer in $\mathrm{SrMnBi}_{2}$. (c) Energy dispersions near the Dirac point along parallel and perpendicular directions to the $\Gamma-M$ symmetry line in $\mathrm{SrMnBi}_{2}[31$ ]. Reprinted with permission from Park et al.[31]. Copyright 2011 by American Physics Society.

ing a transition from single-vortex dominated pinning to a small bundle pinning. This indicates a relatively $2 \mathrm{D}$ nature of the superconducting state as that in the 122-system[47]. The critical current density $J_{c}$ for 112 $\mathrm{Ca}_{1-\mathrm{x}} \mathrm{La}_{\mathrm{x}} \mathrm{FeAs}_{2}$ is about $\sim 10^{5} \mathrm{~A} / \mathrm{cm}^{2}$ (see Fig. 11), only weakly dependent on the direction and strength of the applied magnetic field[47, 77]. However, in Sb-doped $\mathrm{Ca}_{0.85} \mathrm{La}_{0.15} \mathrm{Fe}\left(\mathrm{As}_{0.92} \mathrm{Sb}_{0.08}\right)_{2}$, significant improvement in the $J_{c}$ value[101] was measured $\left(\sim 2.2 \times 10^{6} \mathrm{~A} / \mathrm{cm}^{2}\right)$, which upon high energy proton irradiation can be enhaced upto a value of $6.2 \times 10^{6} \mathrm{~A} / \mathrm{cm}^{2}$. The $J_{c}$ value is comparable to that of 11-type $\mathrm{Fe}_{1+y}(\mathrm{Te}, \mathrm{Se})[102]$ suggesting the importance of strong bulk-dominated or artificial defect induced pinning in enhancing the value of $J_{c}$.

\section{Effect of Pressure}

Application of external pressure can be useful for the suppression of the AFM ground state and stabilisation of superconductivity in Fe-based superconductors without introducing substitutional elements or impurities. For $\mathrm{Ca}_{1-\mathrm{x}} \mathrm{La}_{\mathrm{x}} \mathrm{FeAs}_{2}$, Zhou et al. [45] observed that the resistivity went through a two-step decrease with temperature towards the superconducting phase while no effect of pressure was observed on the normal state resistivity. The temperature $\left(T_{1}\right)$ at which the high- $T$ resistance decrease took place was found to be pressure independent. Both the $T_{c}^{\text {onset }}$ and $T_{c}^{\text {zero }}$ indicated a dome-shaped pressure dependence with its maximum at around 1.19 GPa as illustrated in Fig. 12. The pressure coefficients were comparable to that of other pnictide phases [103, 104]. The maximum $T_{c}^{\text {zero }}$ was $38.5 \mathrm{~K}$ at $1.19 \mathrm{GPa}$ which is much higher than the zero-pressure value of $34 \mathrm{~K}$, suggesting that the enhancement of $T_{\mathrm{c}}$ is possible further in the doped 112-compounds via tuning of the As-Fe-As bond angle as result of the Sb substitution. 


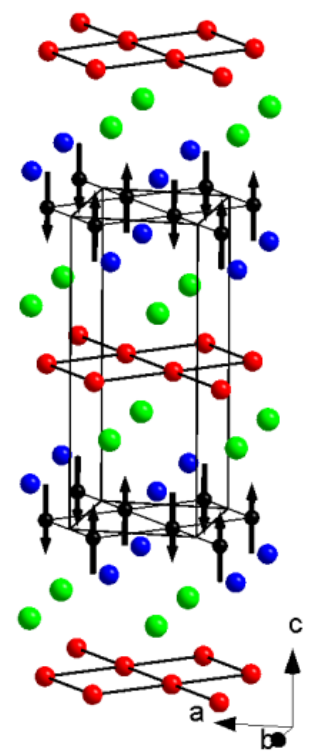

$P 4^{\prime} / n^{\prime} m^{\prime} m$
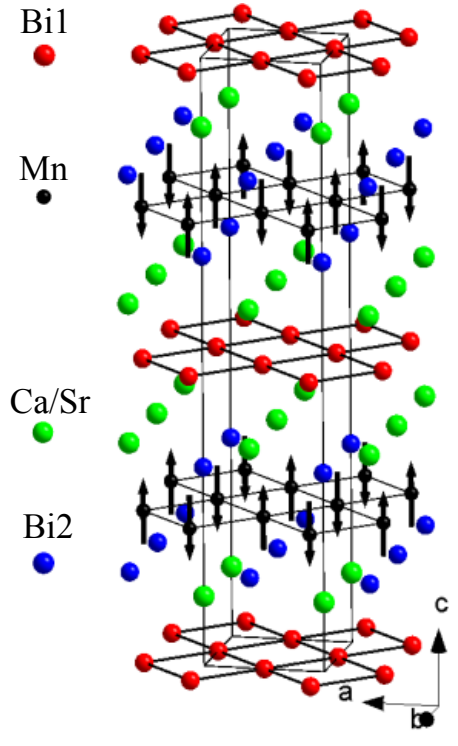

$I 4^{\prime} / m^{\prime} m$ 'm
FIG. 14: Ground state magnetic configurations of $\mathrm{CaMnBi}_{2}$ (left) and $\mathrm{SrMnBi}_{2}$ (right)[63]. Reprinted with permission from Guo et al.[63]. Copyright 2014 by American Physics Society.

\section{Dirac Fermions}

Dirac materials like graphene[105] and topological insulators[106] have attracted significant research interest recently due to their novel quantum mechanical properties that could be useful for quantum computation, nanoelectronics and spintronics. The linear energy dispersion in these materials is governed by the relativistic Dirac equation and the crossing of the linearly dispersed bands at the Dirac point forming a Dirac cone[63]. This configuration suppresses carrier backscattering and enhances electron mobility[31] resulting in novel quantum phenomena like anomalous quantum hall effect and a non-zero Berry phase[105, 106]. The linear energy dispersion also results in large magnetoresistance which increases linearly with magnetic field and does not saturate at higher field[30, 65, 66, 68, 70, 71] as the lowest Landau level can be easily accessed by the Dirac fermions in the quantum limit at moderate applied field. Unlike graphene[105], topological insulators[106] and $d$-wave superconductors[63] with isotropic Dirac cone structures, the range of Dirac materials can be extended further by introducing anisotropy in the Dirac cone for making new electronic devices where electron propagation will be different in different directions from the Dirac point. While various approaches like coupled heterostructures[107], application of strain[108] etc. have been proposed to generate anisotropy[64] in Dirac materials, layered intermetallic compounds like $\mathrm{SrMnBi}_{2}[29-31$, 59-64], $\mathrm{CaMnBi}_{2}[61,63,65,66], \mathrm{LaAgBi}_{2}[60,67-69]$ with 112pnictide structures naturally contain anisotropic Dirac cones (see Fig. 13). The Bi square net layers host the Dirac fermions in such compounds, and the linear en-
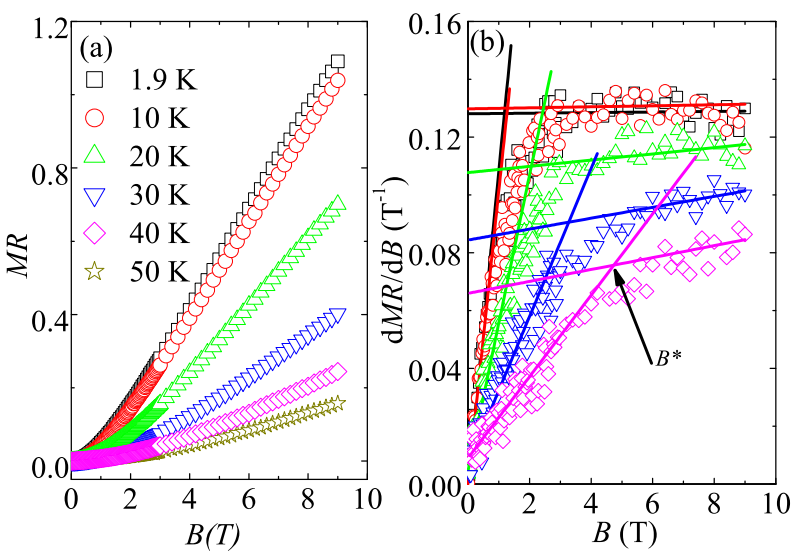

FIG. 15: (a) The magnetic field dependence of the in-plane $M R=\rho(B)-\rho(0)) / \rho(0)$ at various temperatures. (b) First derivative of $M R(\mathrm{~d}(M R) / \mathrm{d} B)$ as function of applied magnetic field at different temperatures. Data at high field was fitted by $M R \sim f(B)+f\left(B^{2}\right)$ and at low field by $M R \sim f\left(B^{2}\right)[30]$. Reprinted with permission from Wang et al.[30]. Copyright 2011 by American Physics Society.

ergy dispersion originates from the crossing of the two Bi- $6 p_{x, y}$ bands [31] which has also been supported by firstprinciples calculations[68] and tight-binding analysis[61]. The tetragonal unit cell of these materials (space group $I 4 / \mathrm{mmm}$ ) is constituted by $\mathrm{Mn}^{-\mathrm{Bi}_{(2)}}$ layers (analogous to the $\mathrm{Fe} P n$ layers) which are separated by $\mathrm{Sr} / \mathrm{Bi}_{(1)}$ layers (Fig. 14) on both sides with the $c$-axis length larger than in other pnictide superconductors[28, 29]. Bi exists in two different valance states in the blocking and tetrahedral layers which is common for 112-phase pnictides.

ARPES measurements on $\mathrm{SrMnBi}_{2}[31]$ revealed the presence of a large circular Fermi surface at the zone centre $(\Gamma$-point) and a needle-like Fermi surface between the $\Gamma$ and $M$ point, as also predicted theoretically. Dirac type dispersion was observed from the needle-shaped Fermi surface along the $\Gamma-M$ line along which the estimated Fermi velocity $\left(v_{F}^{\|}\right)=1.51 \times 10^{6} \mathrm{~m} / \mathrm{s}$ (comparable to that of graphene[105]), while the velocity perpendicular to the $\Gamma-M$ direction $\left(v_{F}^{\perp}\right)$ is $\sim 1.91 \times 10^{5} \mathrm{~m} / \mathrm{s}$. The resulting anisotropy in $v_{F}$ along different directions of the Dirac cone is $v_{F}^{\|} / v_{F}^{\perp} \geq 5$, in agreement with theoretical predictions $[31,62]$. Such anisotropy has been claimed to originate from the different levels of hybridisations in different directions, which along the $\Gamma-M$ line is determined by the overlap between neighbouring $\mathrm{Bi}$ atoms in the square net layer, and in the perpendicular direction is determined by the hybridisation strength between the Sr- $d_{x y, y z}$ and Bi- $p_{x, y}$ orbitals. Jo et al.[59] demonstrated that the interlayer conduction in $\mathrm{SrMnBi}_{2}$ can be valleypolarised under the presence of a tilted magnetic field which also enhances the anisotropy significantly $(\sim 100)$ at high magnetic field.

Unlike graphene with a negligible spin-orbit coupled 


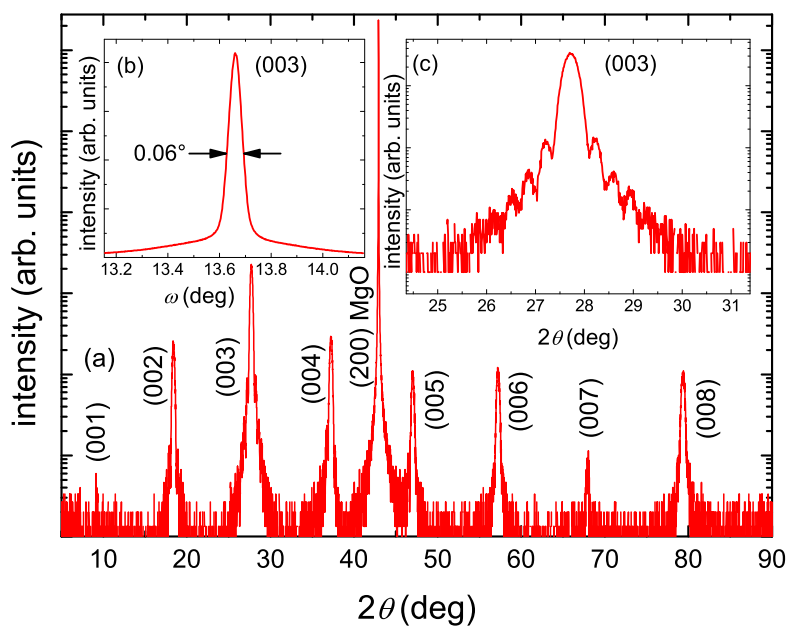

FIG. 16: (a) Out-of-plane $(2 \theta-\omega)$ XRD scans of $\mathrm{LaNi}_{\mathrm{x}} \mathrm{Bi}_{2}$ grown on a $\mathrm{MgO}(100)$ substrate. (b) Typical rocking curve of the (003) peak. (c) Zoom of the (003) peak with Laue fringes[57]. Reprinted with permission from Buckow et al. [57] Copyright 2013 by Institute of Physics.

(SOC) band gap[109], the SOC gap for $\mathrm{SrMnBi}_{2}$ is $\sim$ $40 \mathrm{meV}$ at the Dirac point which can produce a large spin-hall effect[31]. The small effective mass $\left(0.29 m_{e}\right)$, relatively larger carrier mobility $\left(250 \mathrm{~cm}^{2} / \mathrm{Vs}\right)$ and small Fermi surface volume support the existence of Dirac fermions in $\mathrm{SrMnBi}_{2}$. This is further confirmed by the observation of a non-zero Berry phase $(=0.60(9))$ from Shubnikov-de Haas ( $\mathrm{SdH}$ ) oscillations that is expected to be 0.5 for Dirac fermions for a graphene monolayer[105]. As the Bi square net layer hosts Dirac fermions, the SOC gap size could be engineered by replacing $\mathrm{Bi}$ with other pnictogens with lower atomic numbers[71, 110]. Feng et al. [64] observed differences in Dirac cone structures of $\mathrm{CaMnBi}_{2}$ and $\mathrm{SrMnBi}_{2}$ originating from spin-orbit coupling and the arrangement of the $\mathrm{Sr} / \mathrm{Ca}$ ions above and below the Bi square net layer which resulted in a larger gap size in $\mathrm{SrMnBi}_{2}$ than in $\mathrm{CaMnBi}_{2}$.

It was thought earlier that the antiferromagnetic ordering of the Mn-atoms for the cases of $(\mathrm{Sr} / \mathrm{Ca} / \mathrm{Eu}) \mathrm{MnBi}_{2}$ is crucial for the anisotropic behavior, but this has been ruled out after the observation of a Dirac cone like structure close to $E_{F}$ along the $\Gamma-M$ direction in $\mathrm{LaAgBi}_{2}$ [67-69]. However, magnetism seems to be essential for Dirac materials as the long-range magnetic order couples the Dirac fermions which influences the transport behavior [63] as observed from the differences in the magneto-transport behavior of $\mathrm{SrMnBi}_{2}$ and $\mathrm{CaMnBi}_{2}$. Both materials posses Néel type in-plane AFM order, however, the neighboring $\mathrm{MnBi}_{4}$ layers are coupled ferromagnetically in $\mathrm{CaMnBi}_{2}$ and antiferromagnetically in $\mathrm{SrMnBi}_{2}$ which results in a $T_{\mathrm{N}}$ anomaly in $\mathrm{SrMnBi}_{2}$, but not for $\mathrm{SrMnBi}_{2}$ as shown in Fig. 14. The opposite interlayer couplings were claimed to originate from the competition between the AFM super-exchange and FM double-exchange interactions[63].

For the case of $\mathrm{SrMnBi}_{2}$ and $\mathrm{CaMnBi}_{2}, \mathrm{Mn}-$

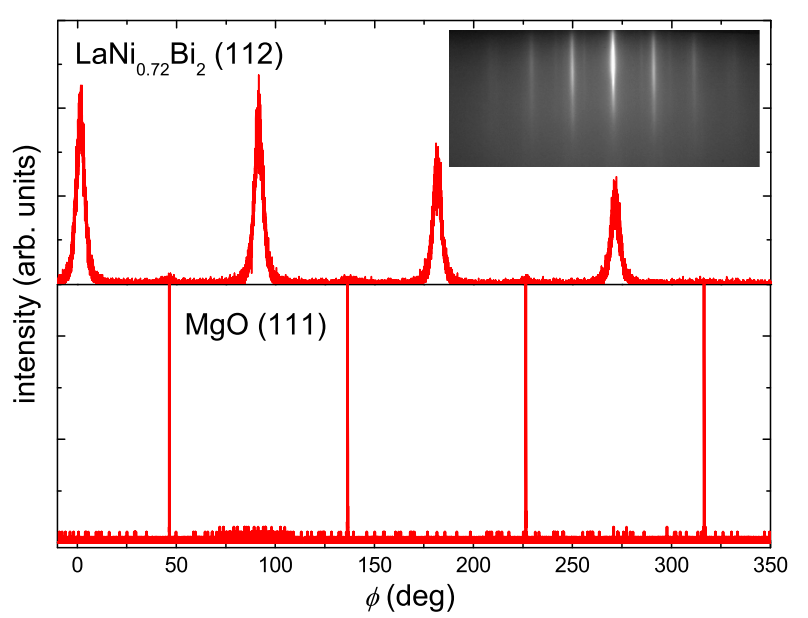

FIG. 17: In-plane X-ray $(\phi)$ scan of $\mathrm{LaNi}_{\mathrm{x}} \mathrm{Bi}_{2}$ grown on $\mathrm{MgO}$ substrate and (inset) RHEED image taken along the (110) direction of $\mathrm{MgO}$ after deposition[57]. Reprinted with permission from Buckow et al.[57]. Copyright 2013 by Institute of Physics.

atoms order antiferromagnetically[29] between $270 \mathrm{~K}$ and $290 \mathrm{~K}[31,68]$ along the $c$-axis, which is different from the case of iso-structural $\mathrm{EuMnBi}_{2}$ where $\mathrm{Mn}$ atoms order at $315 \mathrm{~K}$ and Eu-moments at $T_{\mathrm{N}}=22 \mathrm{~K}[70]$. A significant anisotropy enhancement was observed due to the interaction between $\mathrm{Eu}$ and $\mathrm{Mn}-$ moments that leads to a large increase in magnetoresistance $\sim 650 \%$ at $5 \mathrm{~K}(12 \mathrm{~T})$ which stays unsaturated at the highest applied field of $13 \mathrm{~T}$ at $5 \mathrm{~K}[70]$. Transverse magnetoresistance behavior for $\mathrm{SrMnBi}_{2}, \mathrm{CaMnBi}_{2}, \mathrm{LaAgBi}_{2}, \mathrm{SrZnSb}_{2}[30,65,67$, $68,71]$ goes thorough a semi-classical low field $\left(\sim B^{2}\right)$ dependence to a linear $(\sim B)$ dependence in the highfield limit around a critical field $\left(B^{*}\right)$ as illustrated in Fig. 15. The quadratic temperature dependence of $B^{*}$ can be attributed to Landau level splitting of the linear energy dispersion at high field, with magnetoresistive mobility comparable to that of graphene[30]. Linear magnetic field dependence of the $M R$ supports the existence of a linear dispersion which for $\mathrm{CaMnBi}_{2}$ is $105 \%$ at $10 \mathrm{~T}$ for $H \| c[66]$ and $120 \%$ at $9 \mathrm{~T}(H \perp c)$ and $2 \mathrm{~K}[65]$, for $\mathrm{SrZnSb}_{2} \sim 300 \%$ at $9 \mathrm{~T}$ and $2 \mathrm{~K}[71]$. Large magneto-thermopower was measured in $\mathrm{SrMnBi}_{2}$ with a maximum change of $1600 \%$ at $9 \mathrm{~T}$ and $10 \mathrm{~K}$. The sign of thermopower is positive for $\mathrm{SrMnBi}_{2}$ and negative for $\mathrm{CaMnBi}_{2}$ suggesting that hole and electron-type carriers are responsible for them respectively, although the thermal conductivity stayed independent of magnetic field[60]. The anisotropy and magnetoresistive behavior suggest the possible universal existence of Dirac fermion states in layered compounds with $2 \mathrm{D}$ double sized $\mathrm{Bi}$ square net layers[68].

\section{Thin Films}

High-quality thin films of pnictide compounds will be important for applications, however, they can also do as an useful alternative to those materials where single crys- 


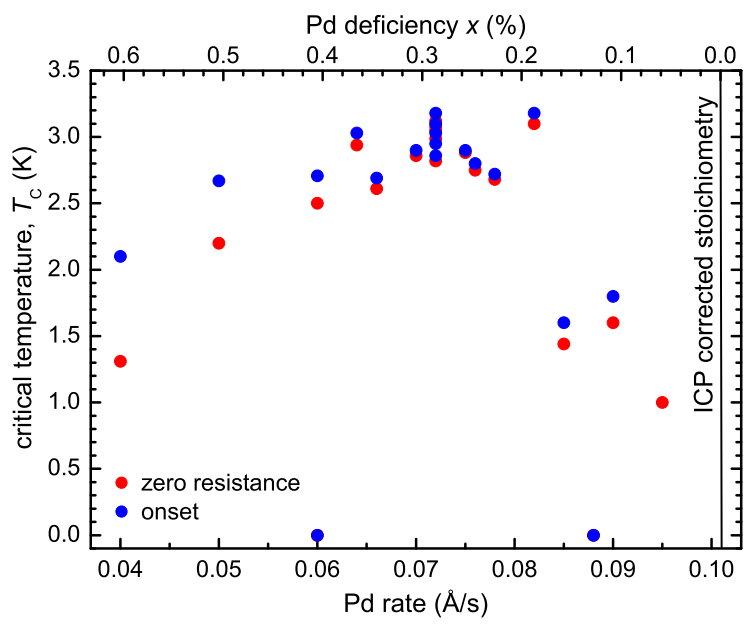

FIG. 18: Phase diagram of $\operatorname{LaPd}_{x} \mathrm{Sb}_{2}$ showing the variation of $T_{\mathrm{c}}$ as function of the $\mathrm{Pd}$ content[55]. Reprinted with permission from Retzlaff et al.[55]. Copyright 2015 by American Physics Society.

tals are still unavailable. Single crystalline phases of pnictide superconductors were grown using MBE and pulsed laser deposition (PLD) showing that thin film techniques are useful for stabilising pnictide compounds (including metastable materials) by using growth kinetics and substrate induced epitaxial strain which is difficult to realize in bulk form. Superconducting films of 122 and 1111phases of pnictides were grown successfully using PLD $[111,112]$ and, in some cases, higher crystallinity and superior superconducting properties were observed in thin films compared to their bulk counterparts $[113,114]$. Recent observation of high- $T_{c}(\sim 65 \mathrm{~K})$ in the 11-structure compound FeSe [26, 115-118] has fuelled interest in superconducting thin film systems. In situ measurements on a monolayer of FeSe grown on $\mathrm{SrTiO}_{3}$ has revealed a $T_{c}>100 \mathrm{~K}[27]$ which is the highest (almost 10 times higher than bulk $T_{c} \sim 10 \mathrm{~K}[12]$ ) in any pnictide system observed so far. The superconductivity in FeSe films has been claimed to originate from interface mode coupling, thus, is most likely not a bulk effect.

\section{A. Growth by Molecular Beam Epitaxy Technique}

The first single-phase superconducting thin film of the 112-phase has been $\mathrm{LaNi}_{x} \mathrm{Bi}_{2}$ grown by MBE[34]. MBE allows to grow a range of materials of various melting points using a combination of electron guns and (hightemperature) effusion cells. This is advantageous in terms of material flexibility, compositional and structural stabilisation as it allows precise doping control. The growth process was carried out in UHV atmosphere (typically at a base pressure $\sim 10^{-9}$ mbar) and typical substrate temperatures between 300 and $600^{\circ} \mathrm{C} . \mathrm{MgO}(100)$ substrates were found to be suitable for epitaxial growth of most of the systems studied[34, 55, 57, 58]. The substrates were pre-annealed at $1000^{\circ} \mathrm{C}$ in atmosphere to improve their surface quality. The growth process was monitored in situ using reflection high energy electron

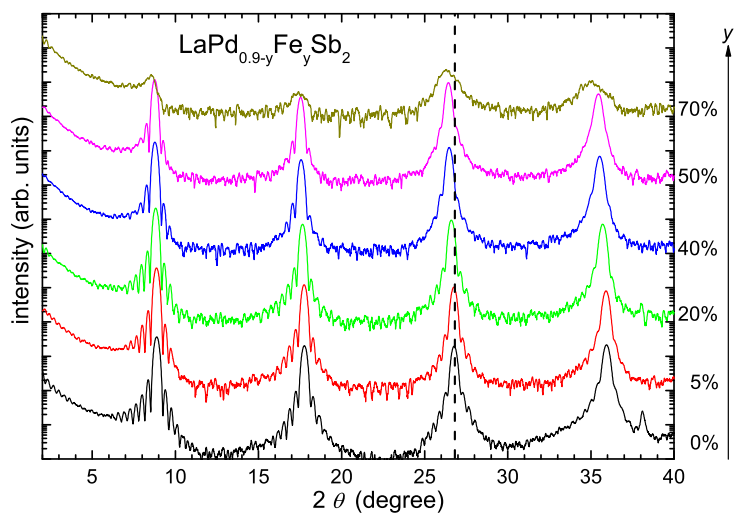

FIG. 19: Out-of-plane $(2 \theta-\omega)$ XRD scans of $\mathrm{LaPd}_{0.9-y} \mathrm{Fe}_{y} \mathrm{Sb}_{2}$ for for various Fe-doping level $0 \leq y \leq 0.7[55]$. Reprinted with permission from Retzlaff et al.[55]. Copyright 2015 by American Physics Society.

diffraction (RHEED).

\section{B. Thin Film Structure}

A list of samples investigated in thin film form along with their growth parameters is shown in Tab. I. For all these materials, the out-of-plane X-ray diffraction (XRD) pattern (see Fig. 16(a)) consisted of a series of (00l) peaks which suggests a ZrCuSiAs-type of crystal structure with the symmetry group $P 4 / \mathrm{nmm}$. The 112-phase thin films were found to be highly $c$-axis oriented and phase pure resulting in some cases in a higher crystalline quality as compared to the bulk synthesised counterpart of the same material. The high crystalline nature of the films was confirmed by the observation of Laue oscillations (see Fig. 16)(c)) with very narrow FWHM typically between 0.03-0.07 (see Fig. 16(b)). The presence of streaky lines in the RHEED pattern in Fig. 17 (inset) indicated the epitaxial nature of the films and smooth nature of the surface. From the X-ray $\phi$-scan in Fig. 17, the fourfold symmetry of the tetragonal structure of the films were observed. In addition to the primary 4-peaks with $90^{\circ}$ separation, another set of 4-peaks with reduced intensities were placed symmetrically between the primary peaks suggesting the presence of two sets of growth domains rotated by $45^{\circ}$ to each other. It was concluded that the peaks with higher intensity correspond to the majority domains which overgrew the minority domains (corresponding to low intensity peaks) which are only present at the substrate/sample interface.

\section{Superconducting and Magnetic Properties of Thin Films}

The $T_{\mathrm{c}}$ was obtained from four probe resistivity measurements where a sharp superconducting transition was observed. At room temperature, all the films had a resistivity in the range of $100 \mu \Omega \mathrm{cm}$. Between $5 \mathrm{~K}$ and $30 \mathrm{~K}$, the resistivity shows metallic behavior that can be fitted using a quadratic temperature dependence $\rho(T)=$ 


\begin{tabular}{lccccc}
\hline \hline Material & $\begin{array}{c}\text { Substrate temperature } \\
\left({ }^{\circ} \mathrm{C}\right)\end{array}$ & $\begin{array}{c}\text { Growth rate } \\
(\AA / \mathrm{s})\end{array}$ & $\begin{array}{c}\text { Lattice constant Lattice constant } \\
\text { in-plane }(\AA)\end{array}$ & $T_{\mathrm{c}}$ \\
& $390-450$ & $\sim 0.5$ & $4.57 \pm 0.1$ & $9.76 \pm 0.01$ & 4.05 \\
\hline $\mathrm{LaNi}_{x} \mathrm{Sb}_{2}[57]$ & $410-440$ & $\sim 0.5$ & $4.565 \pm 0.002$ & $9.64 \pm 0.01$ & 4.05 \\
$\mathrm{CeNi}_{x} \mathrm{Bi}_{2}[34]$ & $400-450$ & $0.25-1.0$ & - & 9.786 & 3.1 \\
$\mathrm{LaNi}_{x} \mathrm{Bi}_{2}[58]$ & $440-520$ & - & $4.52(2)$ & $9.88(5)$ & 3.27 \\
$\mathrm{LaPd}_{x} \mathrm{Sb}_{2}[55]$ & $405-445$ & - & 4.55 & $9.70(9)$ & 3.03 \\
$\mathrm{LaPd}_{x} \mathrm{Bi}_{2}[55]$ &
\end{tabular}

TABLE I: List of 112-type pnictide superconducting thin films and their growth parameters.

$\rho_{0}+A T^{2}$, and from $30 \mathrm{~K}$ tp $300 \mathrm{~K}$, the $\rho(T)$ vs. $T$ dependence is mostly linear. The small residual resistivity ratio[55] $(\sim 1.58)$ indicated a good quality and crystallinity of the samples. Magnetic measurements revealed the presence of Meissner shielding at $T<T_{\mathrm{c}}$ with a significant volume of the superconducting phase. For $\mathrm{LaPd}_{\mathrm{x}} \mathrm{Sb}_{2}$, the lower and upper critical fields were about $10 \mathrm{G}$ and $1.1 \mathrm{~T}$, respectively (with the field applied in out-of-plane direction).

The superconducting phase has been found to depend on the stoichiometric variation of its constituting elements. For $\mathrm{LaPd}_{\mathrm{x}} \mathrm{Sb}_{2}$ within a $\pm 5 \%$ variation of the optimal Sb concentration, $T_{\mathrm{c}}$ stayed almost unaffected, while for larger variations superconductivity vanished associated with a sharp decline in crystallinity. However, for variation in $\mathrm{Pd}$ concentration $T_{\mathrm{c}}$ showed a broad domeshaped behaviour around the mean-value where highest $T_{\mathrm{c}}$ is observed as shown in Fig. 18. The influence of $\mathrm{Fe}$ substitution on the structural behaviour of $\mathrm{LaPd}_{\mathrm{x}} \mathrm{Sb}_{2}$ has been illustrated in Fig. 19. Replacement of Pd by Fe in $\mathrm{LaPd}_{0.9-y} \mathrm{Fe}_{y} \mathrm{Sb}_{2}$ led to a sharp decline in crystallinity for $y>0.5$ indicated by the disappearance of Laue oscillations as shown in Fig. 19, by a shift of the (003) peak position, and also by an increase in the $c$-axis lattice constant. Superconductivity disappeared instantly even on a small level $(\sim 5 \%)$ of Fe substitution in $\mathrm{LaPd}_{\mathrm{x}} \mathrm{Sb}_{2}$, which suggests a conventional $s$-wave nature of the superconducting state as a small amount of ferromagnetic impurities lead to a strong reduction of the BCS density of states. So far, thin films of high- $T_{c}$ 112-pnictides are elusive. Such films could be a new playground for investigating the symmetry of the order parameter and achieving critical temperatures above $77 \mathrm{~K}$.

\section{Conclusion and outlook}

In this review, we have summarised the investigation of superconductivity and Dirac fermions in 112type pnictides. The existence of naturally occurring anisotropic Dirac Cones in the intermetallic 112structure makes them interesting for possible application in nanoelectronics as an alternative to graphene while the large magnetoresistance could be of interest for spintronics. The discovery of high- $T_{\mathrm{c}}$ superconductivity in $\mathrm{Ca}_{1-x} \mathrm{La}_{\mathrm{x}} \mathrm{FeAs}_{2}[16]$ has generated significant research interest in this newly discovered Fe-based super- conducting system. Structurally the 112-phase is quasi2D similar to the 1111-phase, but the presence of a metallic spacer layer and multiple-vacancies of the $P n$-atoms in the neighboring layers are unique properties of this system. Although the parent compound $\mathrm{CaFeAs}_{2}$ has not been synthesised yet, superconductivity can be introduced by $R E$-doping and the $T_{\mathrm{c}}$ has been predicted to go beyond $50 \mathrm{~K}$ for smaller levels of $R E$-content in $\mathrm{Ca}_{1-x} \mathrm{RE}_{x} \mathrm{FeAs}_{2}[18]$. Evidence of a structural and a magnetic phase transition were found both in the nonsuperconducting and superconducting phases[51, 54, 77]. ARPES measurements revealed the presence of three hole like Fermi pockets at the zone centre and two electron like pockets at the zone corner with $3 d$ character similar to the other pnictide systems with moderate nesting between them[16, 53, 78, 79]. In the low- $T_{\mathrm{c}} 112-$ systems, $T_{\mathrm{N}}$ lies below $10 \mathrm{~K}$ and the presence of $\mathrm{Ni}$ vacancies in the $\mathrm{Ni}_{x}$ Bi layer seems to be crucial to stabilize the crystal structure and bulk superconductivity[33]. For $\mathrm{Ca}_{1-\mathrm{x}} \mathrm{La}_{\mathrm{x}} \mathrm{FeAs}_{2}, T_{\mathrm{N}}$ can be enhanced up to $70 \mathrm{~K}[51]$ for a high level of $R E$-doping.

In general, Sb doping helps increasing the in-plane lattice constants. A larger sized pnictogen results in a $T_{\mathrm{c}}$ enhancement with increased superconducting volume fraction for various $R E$-doped $\mathrm{Ca}_{1-x} R E_{x} \mathrm{FeAs}_{2}$ with the so far highest $T_{\mathrm{c}}$ of $47 \mathrm{~K}$. Preliminary measurements suggested a conventional $s$-wave type of pairing symmetry in the 112-phase, although a small amount of $T M$ doping seems to improve the superconducting properties in some cases. The moderate level of anisotropy in the 112phase $\mathrm{Ca}_{1-\mathrm{x}} \mathrm{La}_{\mathrm{x}} \mathrm{FeAs}_{2}$ lies in between the 1111 and 122type pnictide systems, while the upper critical field is significantly higher than for other pnictide phases with comparable critical current density. This makes it interesting from an application perspective as $J_{c}$ can reach $10^{6} \mathrm{~A} / \mathrm{cm}^{2}$ over a large magnetic field range which sounds promising for the fabrication of superconducting tapes and Josephson junctions [47]. Also, it will be interesting to see if the substrate generated strain and interface effects can improve the $T_{\mathrm{c}}$ in a thin film structure similar to the FeSe thin films. Generally, due to the lower level of $R E$-elements the 112-phase will allow significant cost reduction in producing superconducting tapes compared to the 122/1111-pnictide phases. For this purpose, the long-time stability of the 112-compounds will be essential. 


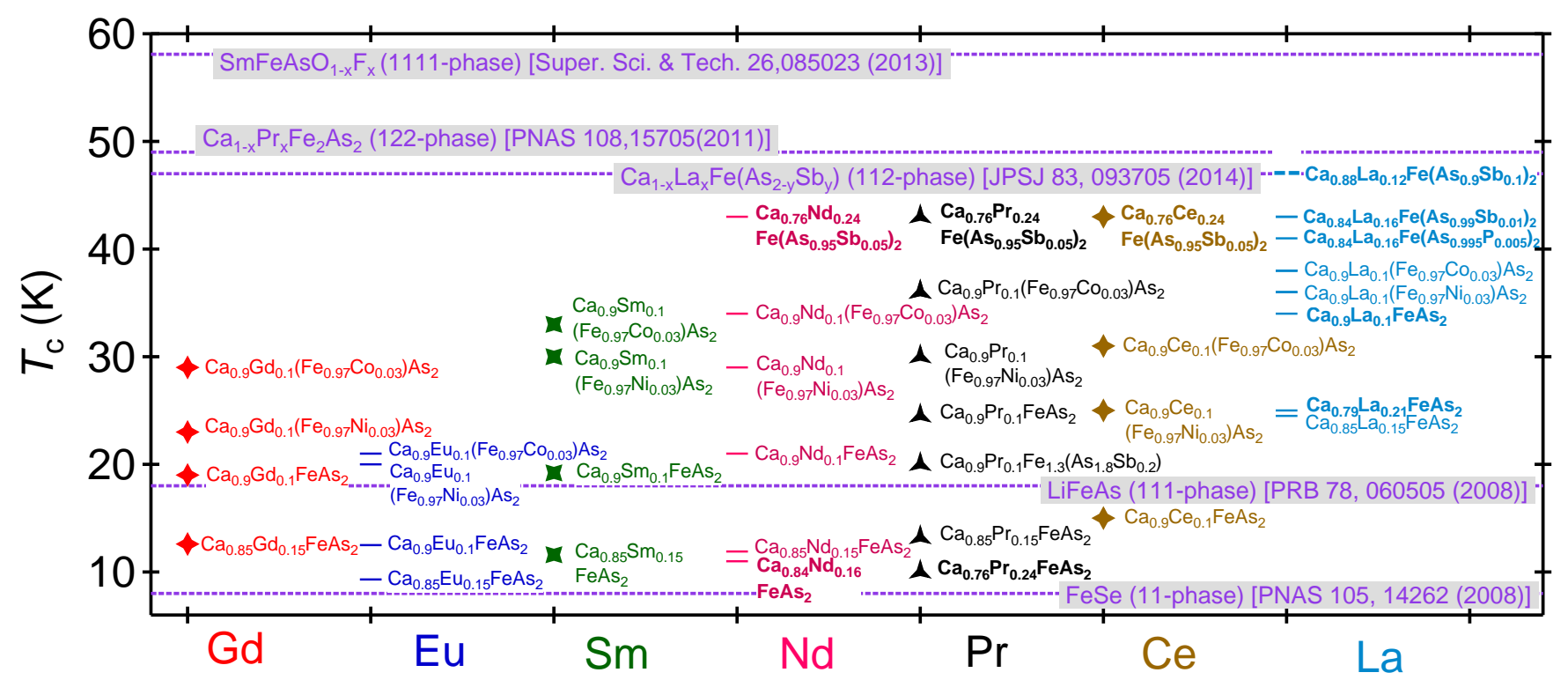

FIG. 20: A phase diagram based on the $T_{\mathrm{c}}$ values of various (Ca,RE)FeAs 2 phases for various RE-elements, arranged in the order of increasing ionic radii under various doping conditions. Symbols indicate $T_{\mathrm{c}}$ values reported so far for various single crystalline (labels in bold fonts) and polycrystalline phases [16-18, 40, 42, 48, 49, 72] ; horizontal dotted lines indicate the maximum $T_{\mathrm{c}}$ values reported for various types of pnictide phases.

Compared to the other pnictide phases, the research work on the 112-system is still at a relatively younger stage and further investigation is needed to understand the potential of this pnictide phase. A list of the $T_{\mathrm{c}}$ 's of various $112-(\mathrm{Ca}, \mathrm{RE}) \mathrm{FeAs}_{2}$ compounds studied so far has been illustrated in Fig. 20. General trend can be found that larger the size of the RE-ion, higher is the $T_{\mathrm{c}}$. It is agreed that further reduction of the RE-content is essential for the further enhancement of $T_{\mathrm{c}}$ in the $\mathrm{Ca}_{1-\mathrm{x}} \mathrm{La}_{\mathrm{x}} \mathrm{FeAs}_{2}$ system which : (a) for the bulk system needs further optimisation of the solid state synthesis techniques and (b) could be possible through various thin film growth processes, but yet to be explored for the $\mathrm{Ca}_{1-\mathrm{x}} \mathrm{La}_{\mathrm{x}} \mathrm{FeAs}_{2}$ system. Scattering techniques like $\mu \mathrm{SR}$ $[119,120]$ and Small Angle Neutron Scattering (SANS) could be useful to explore various parts of the phase diagram : (a) to understand the microscopic presence of magnetism and superconductivity and (b) nature of the vortex lattice symmetry and the vortex phase diagram. It can be expected that the availability of good quality and larger sized single crystals and thin films will allow diverse investigation in this system. Understanding the exact role of $\mathrm{Sb}$ on the enhancement of $T_{\mathrm{c}}$ will help achieving higher $T_{\mathrm{c}}$ in future. In an ideal case, Sb could replace As completely in superconducting 112-compounds with $T_{\mathrm{c}}$ above $77 \mathrm{~K}$ paving the way for a sustainable use of pnictide superconductors in applications.

\section{Acknowledgement}

This work was supported by the Deutsche Forschungsgemeinschaft (DFG) through Grant No. SPP 1458 (LA 560/10-2). We would also like to thank A. Buckow, P. Komissinskiy, K. Kupka, J. Kurian, and R. Retzlaff for their contributions to this project.

\section{References}

[1] Y. Kamihara, M. Hiramatsu, Hidenoriand Hirano, R. Kawamura, H. Yanagi, T. Kamiya, and H. Hosono, Journal of the American Chemical Society 128, 10012 (2006).

[2] Y. Kamihara, T. Watanabe, M. Hirano, , and H. Hosono, Journal of the American Chemical Society 130, 3296 (2008).

[3] H. Takahashi, K. Igawa, K. Arii, Y. Kamihara, M. Hi- rano, and H. Hosono, Nature 453, 376 (2008).

[4] H. Hosono, K. Tanabe, E. Takayama-Muromachi, H. Kageyama, S. Yamanaka, H. Kumakura, M. Nohara, H. Hiramatsu, and S. Fujitsu, Science and Technology of Advanced Materials 16, 033503 (2015).

[5] X. H. Chen, T. Wu, G. Wu, R. H. Liu, H. Chen, and D. F. Fang, Nature 453, 761 (2008).

[6] R. H. Liu, G. Wu, T. Wu, D. F. Fang, H. Chen, S. Y. 
Li, K. Liu, Y. L. Xie, X. F. Wang, R. L. Yang, et al., Phys. Rev. Lett. 101, 087001 (2008).

[7] C. Wang, L. Li, S. Chi, Z. Zhu, Z. Ren, Y. Li, Y. Wang, X. Lin, Y. Luo, S. Jiang, et al., EPL (Europhysics Letters) 83, 67006 (2008).

[8] Z.-A. Ren, G.-C. Che, X.-L. Dong, J. Yang, W. Lu, W. Yi, X.-L. Shen, Z.-C. Li, L.-L. Sun, F. Zhou, et al., EPL (Europhysics Letters) 83, 17002 (2008).

[9] T. Hanna, Y. Muraba, S. Matsuishi, N. Igawa, K. Kodama, S.-i. Shamoto, and H. Hosono, Phys. Rev. B 84, 024521 (2011).

[10] M. Fujioka, S. J. Denholme, T. Ozaki, H. Okazaki, K. Deguchi, S. Demura, H. Hara, T. Watanabe, H. Takeya, T. Yamaguchi, et al., Superconductor Science and Technology 26, 085023 (2013).

[11] M. Rotter, M. Tegel, and D. Johrendt, Phys. Rev. Lett. 101, 107006 (2008).

[12] F.-C. Hsu, J.-Y. Luo, K.-W. Yeh, T.-K. Chen, T.-W. Huang, P. M. Wu, Y.-C. Lee, Y.-L. Huang, Y.-Y. Chu, D.-C. Yan, et al., Proceedings of the National Academy of Sciences 105, 14262 (2008).

[13] J. H. Tapp, Z. Tang, B. Lv, K. Sasmal, B. Lorenz, P. C. W. Chu, and A. M. Guloy, Phys. Rev. B 78, 060505 (2008).

[14] X. Wang, Q. Liu, Y. Lv, W. Gao, L. Yang, R. Yu, F. Li, and C. Jin, Solid State Communications 148, 538 (2008), ISSN 0038-1098.

[15] M. J. Pitcher, D. R. Parker, P. Adamson, S. J. C. Herkelrath, A. T. Boothroyd, R. M. Ibberson, M. Brunelli, and S. J. Clarke, Chem. Commun. pp. 5918-5920 (2008).

[16] N. Katayama, K. Kudo, S. Onari, T. Mizukami, K. Sugawara, Y. Sugiyama, Y. Kitahama, K. Iba, K. Fujimura, N. Nishimoto, et al., Journal of the Physical Society of Japan 82, 123702 (2013).

[17] K. Kudo, T. Mizukami, Y. Kitahama, D. Mitsuoka, K. Iba, K. Fujimura, N. Nishimoto, Y. Hiraoka, and M. Nohara, Journal of the Physical Society of Japan 83, 025001 (2014).

[18] K. Kudo, Y. Kitahama, K. Fujimura, T. Mizukami, H. Ota, and M. Nohara, Journal of the Physical Society of Japan 83, 093705 (2014).

[19] A. Gurevich, Reports on Progress in Physics 74, 124501 (2011).

[20] P. J. W. Moll, R. Puzniak, F. Balakirev, K. Rogacki, J. Karpinski, N. D. Zhigadlo, and B. Batlogg, Nat Mater 9, 628 (2010).

[21] A. Chubukov, Annual Review of Condensed Matter Physics 3, 57 (2012).

[22] D. C. Johnston, Advances in Physics 59, 803 (2010).

[23] C.-H. Lee, A. Iyo, H. Eisaki, H. Kito, M. T. FernandezDiaz, T. Ito, K. Kihou, H. Matsuhata, M. Braden, and K. Yamada, Journal of the Physical Society of Japan 77, 083704 (2008).

[24] A. Taylor, Magnetic Dynamics in Iron-based Superconductors Probed by Neutron Spectroscopy (University of Oxford, UK, 2013).

[25] P. M. Aswathy, J. B. Anooja, P. M. Sarun, and
U. Syamaprasad, Superconductor Science and Technology 23, 073001 (2010).

[26] W. Qing-Yan, L. Zhi, Z. Wen-Hao, Z. Zuo-Cheng, Z. Jin-Song, L. Wei, D. Hao, O. Yun-Bo, D. Peng, C. Kai, et al., Chinese Physics Letters 29, 037402 (2012).

[27] J.-F. Ge, Z.-L. Liu, C. Liu, C.-L. Gao, D. Qian, Q.-K. Xue, Y. Liu, and J.-F. Jia, Nat Mater 14, 285 (2015).

[28] J. H. Shim, K. Haule, and G. Kotliar, Phys. Rev. B 79, 060501 (2009).

[29] J. K. Wang, L. L. Zhao, Q. Yin, G. Kotliar, M. S. Kim, M. C. Aronson, and E. Morosan, Phys. Rev. B 84, 064428 (2011).

[30] K. Wang, D. Graf, H. Lei, S. W. Tozer, and C. Petrovic, Phys. Rev. B 84, 220401 (2011).

[31] J. Park, G. Lee, F. Wolff-Fabris, Y. Y. Koh, M. J. Eom, Y. K. Kim, M. A. Farhan, Y. J. Jo, C. Kim, J. H. Shim, et al., Phys. Rev. Lett. 107, 126402 (2011).

[32] P. F. S. Rosa, C. B. R. Jesus, C. Adriano, Z. Fisk, and P. G. Pagliuso, Journal of Physics: Conference Series 592, 012063 (2015).

[33] H. Mizoguchi, S. Matsuishi, M. Hirano, M. Tachibana, E. Takayama-Muromachi, H. Kawaji, and H. Hosono, Phys. Rev. Lett. 106, 057002 (2011).

[34] A. Buckow, K. Kupka, R. Retzlaff, J. Kurian, and L. Alff, Applied Physics Letters 101, 162602 (2012).

[35] M. H. Jung, A. H. Lacerda, and T. Takabatake, Phys. Rev. B 65, 132405 (2002).

[36] K. Kodama, S. Wakimoto, N. Igawa, S. Shamoto, H. Mizoguchi, and H. Hosono, Phys. Rev. B 83, 214512 (2011).

[37] A. Thamizhavel, A. Galatanu, E. Yamamoto, T. Okubo, M. Yamada, K. Tabata, T. C. Kobayashi, N. Nakamura, K. Sugiyama, K. Kindo, et al., Journal of the Physical Society of Japan 72, 2632 (2003).

[38] H. Mizoguchi, T. Kamiya, and H. Hosono, Solid State Communications 152, 666 (2012), ISSN 0038-1098, special Issue on Iron-based Superconductors.

[39] X. Lin, W. E. Straszheim, S. L. Budko, and P. C. Canfield, Journal of Alloys and Compounds 554, 304 (2013), ISSN 0925-8388.

[40] H. Yakita, H. Ogino, T. Okada, A. Yamamoto, K. Kishio, T. Tohei, Y. Ikuhara, Y. Gotoh, H. Fujihisa, K. Kataoka, et al., Journal of the American Chemical Society 136, 846 (2014).

[41] T. Okada, H. Ogino, H. Yakita, A. Yamamoto, K. Kishio, and J. Shimoyama, Physica C: Superconductivity 505, 1 (2014).

[42] H. Yakita, H. Ogino, A. Sala, T. Okada, A. Yamamoto, K. Kishio, A. Iyo, H. Eisaki, and J. Shimoyama, Physica C: Superconductivity and its Applications 518, 14 (2015), proceedings of the 27th International Symposium on Superconductivity.

[43] N. Katayama, K. Sugawara, A. Nakano, S. Kitou, Y. Sugiyama, N. Kawaguchi, H. Ito, T. Higuchi, T. Fujii, and H. Sawa, Physica C: Superconductivity and its Applications 518, 10 (2015), proceedings of the 27th International Symposium on Superconductivity. 
[44] B. Joseph, C. Marini, N. Demitri, F. Capitani, A. Bernasconi, W. Zhou, X. Xing, and Z. Shi, Superconductor Science and Technology 28, 092001 (2015).

[45] W. Zhou, X. Z. Xing, X. Zhou, M. X. Xu, and Z. X. Shi, EPL (Europhysics Letters) 109, 37005 (2015).

[46] H. Yakita, H. Ogino, A. Sala, T. Okada, A. Yamamoto, K. Kishio, A. Iyo, H. Eisaki, and J. ichi Shimoyama, Superconductor Science and Technology 28, 065001 (2015).

[47] W. Zhou, J. Zhuang, F. Yuan, X. Li, X. Xing, Y. Sun, and Z. Shi, Applied Physics Express 7, 063102 (2014).

[48] A. Sala, H. Yakita, H. Ogino, T. Okada, A. Yamamoto, K. Kishio, S. Ishida, A. Iyo, H. Eisaki, M. Fujioka, et al., Applied Physics Express 7, 073102 (2014).

[49] X. Xing, W. Zhou, B. Xu, N. Li, Y. Sun, Y. Zhang, and Z. Shi, Journal of the Physical Society of Japan 84, 075001 (2015).

[50] M. Y. Li, Z. T. Liu, W. Zhou, H. F. Yang, D. W. Shen, W. Li, J. Jiang, X. H. Niu, B. P. Xie, Y. Sun, et al., Phys. Rev. B 91, 045112 (2015).

[51] S. Kawasaki, T. Mabuchi, S. Maeda, T. Adachi, T. Mizukami, K. Kudo, M. Nohara, and G.-q. Zheng, Phys. Rev. B 92, 180508 (2015).

[52] Z.-H. Liu, T. K. Kim, A. Sala, H. Ogino, J. Shimoyama, B. Bchner, and S. V. Borisenko, Applied Physics Letters 106, 052602 (2015).

[53] Y.-N. Huang, X.-L. Yu, D.-Y. Liu, and L.-J. Zou, Journal of Applied Physics 117, 17E113 (2015).

[54] S. Jiang, C. Liu, H. Cao, T. Birol, J. M. Allred, W. Tian, L. Liu, K. Cho, M. J. Krogstad, J. Ma, et al., Phys. Rev. B 93, 054522 (2016).

[55] R. Retzlaff, A. Buckow, P. Komissinskiy, S. Ray, S. Schmidt, H. Mühlig, F. Schmidl, P. Seidel, J. Kurian, and L. Alff, Phys. Rev. B 91, 104519 (2015).

[56] F. Han, C. D. Malliakas, C. C. Stoumpos, M. Sturza, H. Claus, D. Y. Chung, and M. G. Kanatzidis, Phys. Rev. B 88, 144511 (2013).

[57] A. Buckow, R. Retzlaff, J. Kurian, and L. Alff, Superconductor Science and Technology 26, 015014 (2013).

[58] J. Kurian, A. Buckow, R. Retzlaff, and L. Alff, Physica C: Superconductivity 484, 171 (2013).

[59] Y. J. Jo, J. Park, G. Lee, M. J. Eom, E. S. Choi, J. H. Shim, W. Kang, and J. S. Kim, Phys. Rev. Lett. 113, 156602 (2014).

[60] K. Wang, L. Wang, and C. Petrovic, Applied Physics Letters 100, 112111 (2012).

[61] G. Lee, M. A. Farhan, J. S. Kim, and J. H. Shim, Phys. Rev. B 87, 245104 (2013).

[62] L.-L. Jia, Z.-H. Liu, Y.-P. Cai, T. Qian, X.-P. Wang, H. Miao, P. Richard, Y.-G. Zhao, Y. Li, D.-M. Wang, et al., Phys. Rev. B 90, 035133 (2014).

[63] Y. F. Guo, A. J. Princep, X. Zhang, P. Manuel, D. Khalyavin, I. I. Mazin, Y. G. Shi, and A. T. Boothroyd, Phys. Rev. B 90, 075120 (2014).

[64] Y. Feng, Z. Wang, C. Chen, Y. Shi, Z. Xie, H. Yi, A. Liang, S. He, J. He, Y. Peng, et al., Scientific Reports 4, $5385 \mathrm{EP}$ (2014).

[65] K. Wang, D. Graf, L. Wang, H. Lei, S. W. Tozer, and
C. Petrovic, Phys. Rev. B 85, 041101 (2012).

[66] J. B. He, D. M. Wang, and G. F. Chen, Applied Physics Letters 100, 112405 (2012).

[67] K. Wang and C. Petrovic, Phys. Rev. B 86, 155213 (2012).

[68] K. Wang, D. Graf, and C. Petrovic, Phys. Rev. B 87, 235101 (2013).

[69] X. Shi, P. Richard, K. Wang, M. Liu, C. E. Matt, N. Xu, R. S. Dhaka, Z. Ristic, T. Qian, Y.-F. Yang, et al., Arxiv: 1512.04187 (2015).

[70] A. F. May, M. A. McGuire, and B. C. Sales, Phys. Rev. B 90, 075109 (2014).

[71] K. Wang and C. Petrovic, Applied Physics Letters 101, 152102 (2012).

[72] H. Yakita, H. Ogino, A. Sala, T. Okada, A. Yamamoto, K. Kishio, A. Iyo, H. Eisaki, and J. ichi Shimoyama, Superconductor Science and Technology 28, 065001 (2015).

[73] F. Han, X. Wan, D. Phelan, C. C. Stoumpos, M. Sturza, C. D. Malliakas, Q. Li, T.-H. Han, Q. Zhao, D. Y. Chung, et al., Phys. Rev. B 92, 045112 (2015).

[74] J. W. Harter, H. Chu, S. Jiang, N. Ni, and D. Hsieh, Phys. Rev. B 93, 104506 (2016).

[75] S. Matsuishi, Y. Inoue, T. Nomura, H. Yanagi, M. Hirano, and H. Hosono, Journal of the American Chemical Society 130, 14428 (2008).

[76] B. Joseph, A. Iadecola, A. Bernasconi, P. Rispoli, N. Demitri, X. Xing, W. Zhou, and Z. Shi, Journal of Physics and Chemistry of Solids 84, 24 (2015).

[77] F. Caglieris, A. Sala, M. Fujioka, F. Hummel, I. Pallecchi, G. Lamura, D. Johrendt, Y. Takano, S. Ishida, A. Iyo, et al., APL Mater. 4, 020702 (2016).

[78] L. Xu, L. De-Fa, Z. Lin, G. Qi, M. Qing-Ge, C. DongYun, S. Bing, Y. He-Mian, H. Jian-Wei, H. Jun-Feng, et al., Chinese Physics Letters 30, 127402 (2013).

[79] G. Wang, X. Shi, L. Zhang, and X. Yi, Solid State Communications 200, 61 (2014).

[80] I. I. Mazin, D. J. Singh, M. D. Johannes, and M. H. Du, Phys. Rev. Lett. 101, 057003 (2008).

[81] D. J. Singh and M.-H. Du, Phys. Rev. Lett. 100, 237003 (2008).

[82] H. Ding, P. Richard, K. Nakayama, K. Sugawara, T. Arakane, Y. Sekiba, A. Takayama, S. Souma, T. Sato, T. Takahashi, et al., EPL (Europhysics Letters) 83, 47001 (2008).

[83] X. Wu, C. Le, Y. Liang, S. Qin, H. Fan, and J. Hu, Phys. Rev. B 89, 205102 (2014).

[84] X. Wu, S. Qin, Y. Liang, C. Le, H. Fan, and J. Hu, Phys. Rev. B 91, 081111 (2015).

[85] Y. Nagai, H. Nakamura, M. Machida, and K. Kuroki, Journal of the Physical Society of Japan 84, 093702 (2015).

[86] S. Thirupathaiah, T. Stürzer, V. B. Zabolotnyy, D. Johrendt, B. Büchner, and S. V. Borisenko, Phys. Rev. B 88, 140505 (2013).

[87] A. Charnukha, S. Thirupathaiah, V. B. Zabolotnyy, B. Büchner, N. D. Zhigadlo, B. Batlogg, A. N. Yaresko, 
and S. V. Borisenko, Scientific Reports 5, 10392 EP (2015).

[88] F. Steglich, J. Aarts, C. D. Bredl, W. Lieke, D. Meschede, W. Franz, and H. Schäfer, Phys. Rev. Lett. 43, 1892 (1979).

[89] H. Kito, H. Eisaki, and A. Iyo, Journal of the Physical Society of Japan 77, 063707 (2008).

[90] M. Nicklas, O. Stockert, T. Park, K. Habicht, K. Kiefer, L. D. Pham, J. D. Thompson, Z. Fisk, and F. Steglich, Phys. Rev. B 76, 052401 (2007).

[91] N. Fujiwara, S. Tsutsumi, S. Iimura, S. Matsuishi, H. Hosono, Y. Yamakawa, and H. Kontani, Phys. Rev. Lett. 111, 097002 (2013).

[92] M. Hiraishi, S. Iimura, K. M. Kojima, J. Yamaura, H. Hiraka, K. Ikeda, P. Miao, Y. Ishikawa, S. Torii, M. Miyazaki, et al., Nat Phys 10, 300 (2014).

[93] R. Sakurai, N. Fujiwara, N. Kawaguchi, Y. Yamakawa, H. Kontani, S. Iimura, S. Matsuishi, and H. Hosono, Phys. Rev. B 91, 064509 (2015).

[94] S. R. Saha, N. P. Butch, T. Drye, J. Magill, S. Ziemak, K. Kirshenbaum, P. Y. Zavalij, J. W. Lynn, and J. Paglione, Phys. Rev. B 85, 024525 (2012).

[95] K. Kudo, K. Iba, M. Takasuga, Y. Kitahama, J.-i. Matsumura, M. Danura, Y. Nogami, and M. Nohara, Scientific Reports 3, 1478 EP (2013).

[96] B. Lv, L. Deng, M. Gooch, F. Wei, Y. Sun, J. K. Meen, Y.-Y. Xue, B. Lorenz, and C.-W. Chu, Proceedings of the National Academy of Sciences 108, 15705 (2011).

[97] A. S. Sefat, R. Jin, M. A. McGuire, B. C. Sales, D. J. Singh, and D. Mandrus, Phys. Rev. Lett. 101, 117004 (2008).

[98] A. S. Sefat, A. Huq, M. A. McGuire, R. Jin, B. C. Sales, D. Mandrus, L. M. D. Cranswick, P. W. Stephens, and K. H. Stone, Phys. Rev. B 78, 104505 (2008).

[99] J. Jaroszynski, F. Hunte, L. Balicas, Y.-j. Jo, I. Raičević, A. Gurevich, D. C. Larbalestier, F. F. Balakirev, L. Fang, P. Cheng, et al., Phys. Rev. B 78, 174523 (2008).

[100] H. Q. Yuan, J. Singleton, F. F. Balakirev, S. A. Baily, G. F. Chen, J. L. Luo, and N. L. Wang, Nature 457, 565 (2009).

[101] A. Park, A. Mine, T. Yamada, F. Ohtake, H. Akiyama, Y. Sun, S. Pyon, T. Tamegai, Y. Kitahama, T. Mizukami, et al., Superconductor Science and Technology 29, 055006 (2016).

[102] Y. Sun, T. Taen, Y. Tsuchiya, Q. Ding, S. Pyon, Z. Shi, and T. Tamegai, Applied Physics Express 6, 043101 (2013).
[103] K. Ahilan, F. L. Ning, T. Imai, A. S. Sefat, M. A. McGuire, B. C. Sales, and D. Mandrus, Phys. Rev. B 79, 214520 (2009).

[104] A. F. Wang, Z. J. Xiang, J. J. Ying, Y. J. Yan, P. Cheng, G. J. Ye, X. G. Luo, and X. H. Chen, New Journal of Physics 14, 113043 (2012).

[105] Y. Zhang, Y.-W. Tan, H. L. Stormer, and P. Kim, Nature 438, 201 (2005).

[106] M. Z. Hasan and C. L. Kane, Rev. Mod. Phys. 82, 3045 (2010).

[107] V. Pardo and W. E. Pickett, Phys. Rev. Lett. 102, 166803 (2009).

[108] S.-M. Choi, S.-H. Jhi, and Y.-W. Son, Phys. Rev. B 81, 081407 (2010).

[109] H. Min, J. E. Hill, N. A. Sinitsyn, B. R. Sahu, L. Kleinman, and A. H. MacDonald, Phys. Rev. B 74, 165310 (2006).

[110] M. A. Farhan, G. Lee, and J. H. Shim, Journal of Physics: Condensed Matter 26, 042201 (2014).

[111] H. Hiramatsu, T. Katase, T. Kamiya, M. Hirano, and H. Hosono, Applied Physics Express 1, 101702 (2008).

[112] H. Hiramatsu, T. Katase, T. Kamiya, M. Hirano, and H. Hosono, Applied Physics Express 1, 101702 (2008).

[113] K. Iida, J. Hnisch, R. Hhne, F. Kurth, M. Kidszun, S. Haindl, J. Werner, L. Schultz, and B. Holzapfel, Applied Physics Letters 95, 192501 (2009).

[114] T. Katase, H. Hiramatsu, T. Kamiya, and H. Hosono, Applied Physics Express 3, 063101 (2010).

[115] D. Liu, W. Zhang, D. Mou, J. He, Y.-B. Ou, Q.-Y. Wang, Z. Li, L. Wang, L. Zhao, S. He, et al., Nat Commun 3, 931 (2012).

[116] S. Tan, Y. Zhang, M. Xia, Z. Ye, F. Chen, X. Xie, R. Peng, D. Xu, Q. Fan, H. Xu, et al., Nat Mater 12, 634 (2013).

[117] S. He, J. He, W. Zhang, L. Zhao, D. Liu, X. Liu, D. Mou, Y.-B. Ou, Q.-Y. Wang, Z. Li, et al., Nat Mater 12, 605 (2013).

[118] Y. Sun, W. Zhang, Y. Xing, F. Li, Y. Zhao, Z. Xia, L. Wang, X. Ma, Q.-K. Xue, and J. Wang, Scientific Reports 4, $6040 \mathrm{EP}$ (2014).

[119] D. O. G. Heron, S. J. Ray, S. J. Lister, C. M. Aegerter, H. Keller, P. H. Kes, G. I. Menon, and S. L. Lee, Phys. Rev. Lett. 110, 107004 (2013).

[120] S. J. Ray, A. S. Gibbs, S. J. Bending, P. J. Curran, E. Babaev, C. Baines, A. P. Mackenzie, and S. L. Lee, Phys. Rev. B 89, 094504 (2014). 